\title{
A Numerical Study of Eigenvalues of the Hyperbolic Laplacian for Polyhedra with One Cusp
}

\author{
Fritz Grunewald and Wolfgang Huntebrinker
}

\section{CONTENTS}

1. Introduction

2. The Eigenvalue Problem

3. Some Discontinuous Groups and Their Fundamental Domains

\section{Discretization}

5. Numerical Methods

6. Symmetries of Eigenfunctions

7. Numerical Computations

8. Tabulation of Results

References
Huntebrinker's research was supported by the Deutsche Forschungsgemeinschaft.
Let $\mathbb{H}_{3}$ be three-dimensional hyperbolic space and $\Gamma$ a group of isometries of $\mathbb{H}_{3}$ that acts discontinuously on $\mathbb{H}_{3}$ and that has a fundamental domain of finite hyperbolic volume. The Laplace operator $-\Delta$ of $\mathbb{H}_{3}$ gives rise to a positive, essentially selfadjoint operator on $L^{2}\left(\Gamma \backslash \mathbb{H}_{3}\right)$. The nature of its discrete spectrum dspec $\Gamma$ is still not well understood if $\Gamma$ is not cocompact.

This paper contains a report on a numerical study of $\operatorname{dspec} \Gamma$ for various noncocompact groups $\Gamma$. Particularly interesting are the results for some nonarithmetic groups $\Gamma$.

\section{INTRODUCTION}

For an integer $n \geq 2$, let $\mathbb{H}_{n}$ be $n$-dimensional hyperbolic space, that is, the connected and simply connected Riemannian $n$-manifold of constant curvature -1 . Denote by $d s$ the line element of $\mathbb{H}_{n}$, by $d v$ its volume form, and by $\Delta$ its Laplacian. Let Iso $\mathbb{H}_{n}$ be the group of isometries of $\mathbb{H}_{n}$, and $\mathrm{Iso}^{+} \mathbb{H}_{n}$ the subgroup of orientation-preserving isometries. If $\Gamma \subset$ Iso $\mathbb{H}_{n}$ is a subgroup, let $\Gamma^{+}:=\Gamma \cap \mathrm{Iso}^{+} \mathbb{H}_{n}$.

Throughout this article we will assume that $\Gamma$ is a group that acts discontinuously on $\mathbb{H}_{n}$, and that the quotient space $\Gamma^{+} \backslash \mathbb{H}_{n}$ has finite hyperbolic volume. Such a group is called cofinite. A cofinite group $\Gamma$ admits a fundamental domain $\mathcal{F}_{\Gamma} \subset \mathbb{H}_{n}$ that is a hyperbolic polyhedron, bounded if and only if $\Gamma^{+} \backslash \mathbb{H}_{n}$ is compact.

Since $\Gamma$ normalizes $\Gamma^{+}$, it acts by translation on $L^{2}\left(\Gamma^{+} \backslash \mathbb{H}_{n}, d v\right)$. Let $L^{2}(\Gamma)=L^{2}\left(\Gamma^{+} \backslash \mathbb{H}_{n}, d v\right)^{\Gamma}$ be the closed subspace of $\Gamma$-invariant functions.

It is known that the Laplace operator $-\Delta$ defined on a suitable domain in $L^{2}(\Gamma)$ has a unique 
positive selfadjoint extension to an operator, also called $-\Delta$, on $L^{2}(\Gamma)$. The spectrum spec $\Gamma$ of $-\Delta$ on $L^{2}(\Gamma)$ decomposes into discrete and continuous parts:

$$
\operatorname{spec} \Gamma=\operatorname{dspec} \Gamma \cup \operatorname{cspec} \Gamma .
$$

The continuous spectrum cspec $\Gamma$ is empty if $\Gamma \backslash \mathbb{H}_{n}$ is compact (such groups are called cocompact) and otherwise the union of finitely many intervals

$$
\left[\frac{1}{4}(n-1)^{2}, \infty\right) \text {. }
$$

The discrete spectrum contains 0 and is moreover a discrete subset of the nonnegative real numbers. It is an important question how the sets $\operatorname{dspec} \Gamma \subset$ $[0, \infty)$ might look in general. When $\Gamma$ is cocompact, it is known that $\operatorname{dspec} \Gamma$ is infinite and satisfies Weyl's asymptotic law. To formulate this distribution law we define

$$
C(\Gamma):=\frac{(4 \pi)^{-n / 2} \operatorname{vol}\left(\Gamma^{+} \backslash \mathbb{H}_{n}\right)}{\Gamma\left(\frac{1}{2} n+1\right)\left[\Gamma: \Gamma^{+}\right]},
$$

where vol is the hyperbolic volume and $\Gamma(\cdot)$ represents the usual $\Gamma$-function. For a real number $T$ we put

$$
N_{\Gamma}(T):=\left|\left\{\lambda \in \operatorname{dspec} \Gamma: \lambda \leq T^{2}\right\}\right|,
$$

counting eigenvalues with their multiplicities. We say that $\Gamma$ satisfies Weyl's law if

$$
\lim _{T \rightarrow \infty} \frac{N_{\Gamma}(T)}{T^{n}}=C(\Gamma) .
$$

It is also known that cofinite (not cocompact) arithmetic congruence groups $\Gamma$ satisfy Weyl's law [Selberg 1956; Venkov 1977a; 1979; 1981], and that dspec $\Gamma$ is an infinite set if $\Gamma$ is not equal to its normalizer in Iso $\mathbb{H}_{n}$ or if, more generally, $\Gamma$ is not equal to its commensurator in Iso $\mathbb{H}_{n}$ [Venkov 1977b; 1978]. Note that if $\Gamma$ is arithmetic then it is of infinite index in its commensurator.

This being what is known in general, there is the following fascinating story for the case $n=2$. Here $\mathbb{H}_{2}$ is the hyperbolic plane and the groups $\Gamma$ are the classical cofinite Fuchsian groups. After an older conjecture of Roelcke and Selberg that any such group should satisfy Weyl's asymptotic law, a better understanding due to Phillips and Sarnak [1985a; 1985b] led to the conjecture that for generic cofinite but not cocompact groups $\Gamma$ the discrete spectrum dspec $\Gamma$ should only consist of finitely many elements. The new conjecture derives its credibility from Fermi's golden rule, a principle well known in physics: A cofinite group $\Gamma$ with infinite dspec $\Gamma$ might be deformable in a suitable Teichmüller space. A small deformation should then lead to a new group, for which all eigenvalues embedded in the continuous spectrum should have disappeared. The term "generic" is at the moment still somewhat unclear; one hopes it might mean something like an element in general position in a Teichmüller space. The new conjecture has subsequently been heuristically checked. There where also important steps toward a proof: see [Hejhal and Rackner 1992; Sarnak 1986; Sarnak 1990; Wolpert 1994] for the state of the art.

There is the effort of P. Sarnak $[1986 ; 1990]$ to get a conjectural understanding of the situation for more general symmetric spaces. For the spaces $\mathbb{H}_{n}$ with $n \geq 3$ the situation remains difficult to judge. On the one hand there are many nonarithmetic or arithmetic noncongruence cofinite groups. On the other hand a heuristic reasoning using Fermi's golden rule is not possible since, due to Mostow's rigidity theorem, there are no genuine deformations of the groups [Elstrodt et al. $\geq 1996$ ].

This paper reports on a numerical study of the discrete spectrum dspec $\Gamma$ for some cofinite noncocompact groups $\Gamma<\mathrm{Iso}^{+} \mathbb{H}_{3}$ that admit a fundamental domain of a simple nature. We mainly consider fundamental domains that are tetrahedra with exactly one point at infinity or that are simple combinations of such. There are exactly nine tetrahedra with one point at infinity that tesselate $\mathbb{H}_{3}$. These tetrahedra and their reflection groups are described in Section 3.1.

Some of the groups so obtained are arithmetic. To describe them we identify $\mathrm{Iso}^{+} \mathbb{H}_{3}$ with $\mathrm{PSL}_{2}(\mathbb{C})$. If $\mathcal{O}$ is the ring of integers in an imaginary quadratic number field then $\mathrm{PSL}_{2}(\mathcal{O})$ is an arithmetic, 
cofinite, noncocompact subgroup of $\mathrm{PSL}_{2}(\mathbb{C})$. In fact a cofinite, noncocompact subgroup $\Gamma<$ Iso $\mathbb{H}_{3}$ is arithmetic if and only if it is Iso $\mathbb{H}_{3}$-conjugate to a group $\tilde{\Gamma}$ commensurable to one of the groups $\mathrm{PSL}_{2}(\mathcal{O})$. It is a congruence group if $\tilde{\Gamma}$ can be chosen to contain a full congruence group of $\mathrm{PSL}_{2}(\mathcal{O})$.

For example, we were able to treat the groups of imaginary quadratic integers $\mathrm{PSL}_{2}(\mathbb{Z}[a])$ for $a=i$, $a=\sqrt{-2}$, and $a=\frac{1}{2}(-1+\sqrt{-3})$, as well as some groups commensurable to these. Section 8.1 contains tables of the small eigenvalues. Here is a sample of the results.

The group $E_{5}$ from Section 3.6 is noncocompact, nonarithmetic, and equal to its commensurator. We have found the following approximate eigenvalues below 220 :

$$
\begin{aligned}
& 45.1,95,112,118,145, \\
& 163,173,189,196,199,216 ?
\end{aligned}
$$

(The question mark indicates we were not certain from the numerical results that the eigenfunction we obtained for this eigenvalue satisfied the $L^{2}$ condition.)

We also find

$$
C\left(E_{5}\right) \approx 0.002926
$$

and

$$
\frac{N_{E_{5}}(15)}{15^{3}} \approx 0.003259
$$

Considering similar computations in the arithmetic cases this seems to be a reasonable case for Weyl's asymptotic law, which reads in dimension three as follows:

$$
\lim _{T \rightarrow \infty} \frac{N_{\Gamma}(T)}{T^{3}}=C(\Gamma)=\frac{\operatorname{vol}\left(\Gamma^{+} \backslash \mathbb{H}_{n}\right)}{6 \pi^{2}\left[\Gamma: \Gamma^{+}\right]} .
$$

We also mention the reflection group $\Gamma_{0}$ of a tesselating tetrahedron with two points at infinity, given in the notation of Section 3.1 by

$$
\mathcal{T}=\left[60^{\circ}, 30^{\circ}, 90^{\circ} ; 60^{\circ}, 60^{\circ}, 90^{\circ}\right]
$$

This group, too, is noncocompact, nonarithmetic, and equal to its commensurator. We have found the following table of eigenvalues below 187 :

$$
\begin{aligned}
& 32.8,68,70 ?, 76,92,94,116 ?, 124, \\
& 148,150,160 ?, 163,182 ?, 186
\end{aligned}
$$

Since $\Gamma_{0}$ has two points at infinity, the method of computation is more involved and will be described in a later paper [Grunewald and Huntebrinker].

Our computations of eigenvalues use finite element methods. In Sections 4 and 5 we report on the procedure that we have used; more details are given in [Huntebrinker 1995]. Right now we comment only on questions of accuracy and appropriateness of our method. First of all, our results have gone through many compatibility checks, described in [Huntebrinker 1995]. Secondly, in two dimensions, an earlier version of the program reported on here produced very good agreement with results produced by other methods [Huntebrinker 1991].

In three dimensions comparative data are more scarce. There is one published table of eigenvalues [Smotrov and Golovchansky 1991] that was found by a different method. It contains the twelve smallest eigenvalues for $\Gamma=\mathrm{PSL}_{2}(\mathbb{Z}[i])$. Because of an error in one of their corollaries, the authors were only able to catch eigenfunctions that are also invariant by the bigger group $\mathrm{PGL}_{2}(\mathbb{Z}[i])$, considered as a subgroup of $\mathrm{PSL}_{2}(\mathbb{C})$ via the embedding

$$
g \mapsto \frac{g}{\sqrt{\operatorname{det} g}} .
$$

These eigenfunctions are those of our symmetry types $\mathrm{C}$ and D. Taking this into account we get perfect agreement between that table and our results.

Recently we became aware of a list, computed by G. Steil and F. Steiner at the Institute of Theoretical Physics at the University of Hamburg, containing eigenvalues under 900 for $\Gamma=\mathrm{PSL}_{2}(\mathbb{Z}[i])$, with six-digit accuracy. It was computed by a different method, relying on Fourier expansions of the eigenfunctions. Agreement with our results is 
again good. For the smallest eigenvalues we obtain the same accuracy; for larger eigenvalues our results are worse.

There have been reports of significant progress on the high-precision computation of eigenvalues for triangle groups in Iso $\mathbb{H}_{2}$ by the Fourier expansion method [Bogomolny et al. 1992; Bolte et al. 1992; Hejhal 1992; Hejhal and Rackner 1992; Steil 1994]. Although our method does not give the same precision, it has the merit of flexibility. It doesn't use the existence of Hecke operators and is also applicable if $\Gamma$ has more than one cusp. It allowed us to perform an experiment that apparently could not be done by the Fourier expansion methods, as follows.

As mentioned above, Mostow's theorem prevents us from deforming discrete groups of three-dimensional hyperbolic isometries. So we studied what happens if their fundamental tetrahedra were deformed. This can be done by continuously varying angles or edge lengths. We have done computations for an interesting continuous family of such tetrahedra (Section 3.7) that includes fundamental domains. We have fixed boundary conditions (Dirichlet or Neumann) on the bounding planes and looked for exponentially decaying (approaching the infinite vertex) solutions. These results are described in Section 8.2. In the case of full Neumann conditions on the boundary the tables give an interesting picture.

The programs were designed by the second author, and information on them can be found in [Huntebrinker 1995], together with tables of eigenvalues for subgroups of finite index in the groups treated here. Our actual numerical computations were done on IBM RS/6000 machines.

\section{THE EIGENVALUE PROBLEM}

We start by describing the upper half-space model of three-dimensional hyperbolic space. Then we summarize the $L^{2}$-spectral theory of the Laplace operator for certain quotients of hyperbolic space. We also fix some notation to be used in the sequel.

\subsection{The Laplace Operator for Hyperbolic Space}

Let $\mathbb{H}_{3}$ be the upper half-space in $\mathbb{R}^{3}$, that is,

$$
\mathbb{H}_{3}=\left\{(x, y, r) \in \mathbb{R}^{3}: r>0\right\} .
$$

The hyperbolic metric on $\mathbb{H}_{3}$ is given by the line element

$$
d s^{2}=\frac{d x^{2}+d y^{2}+d r^{2}}{r^{2}} .
$$

This line element induces the volume form

$$
d v=\frac{d x d y d r}{r^{3}}
$$

The corresponding Laplace operator is given by

$$
\Delta=r^{2}\left(\frac{\partial^{2}}{\partial x^{2}}+\frac{\partial^{2}}{\partial y^{2}}+\frac{\partial^{2}}{\partial r^{2}}\right)-r \frac{\partial}{\partial r}
$$

[Elstrodt et al. 1987].

We write Iso $\mathbb{H}_{3}$ and Iso ${ }^{+} \mathbb{H}_{3}$ for the group of isometries of $\mathbb{H}_{3}$ and its subgroup consisting of orientation-preserving maps. $\mathrm{Iso}^{+} \mathbb{H}_{3}$ can be identified with the group $\mathrm{PSL}_{2}(\mathbb{C})$ : Thinking of upper half-space as $\mathbb{C} \times \mathbb{R}^{+}$, with $(x, y, r)$ corresponding to $(x+y i, r)$, the action of

$$
\left(\begin{array}{ll}
a & b \\
c & d
\end{array}\right) \in \mathrm{PSL}_{2}(\mathbb{C})
$$

takes $(z, r) \in \mathbb{C} \times \mathbb{R}^{+}$to

$$
\left(\frac{(a z+b)(\bar{c} \bar{z}+\bar{d})+a \bar{c} r^{2}}{|c z+d|^{2}+|c|^{2} r^{2}}, \frac{r}{|c z+d|^{2}+|c|^{2} r^{2}}\right) .
$$

The group Iso $\mathbb{H}_{3}$ is then the extension of $\mathrm{PSL}_{2}(\mathbb{C})$ by the complex reflection

$$
\tau: \mathbb{H}_{3} \rightarrow \mathbb{H}_{3}, \quad \tau:(x, y, r) \mapsto(x,-y, r) .
$$

We consider here subgroups $\Gamma \subseteq$ Iso $\mathbb{H}_{3}$ that act discontinuously on $\mathbb{H}_{3}$. We suppose further that $\Gamma$ admits a (measurable) fundamental domain $\Omega \subset \mathbb{H}_{3}$ of finite hyperbolic volume but that the quotient space $\Gamma \backslash \mathbb{H}_{3}$ is not compact.

Such a $\Gamma$ is known to have a fundamental domain $\Omega=\mathcal{F}_{\Gamma} \subseteq \mathbb{H}_{3}$ that is an unbounded hyperbolic polyhedron. One can choose $\Omega$ to have a boundary consisting of finitely many polygonal pieces of hyperbolic planes. 
For $\Gamma \subset \mathrm{Iso}^{+} \mathbb{H}_{3}$ we write $L^{2}\left(\Gamma \backslash \mathbb{H}_{3}, d v\right)$ for the set of classes of Borel measurable functions $f$ : $\mathbb{H}_{3} \rightarrow \mathbb{R}$ that are $\Gamma$-invariant (that is, satisfy $f \circ \gamma=$ $f$ for all $\gamma \in \Gamma)$ and square-integrable:

$$
\int_{\mathcal{F}_{\Gamma}}|f|^{2} d v<\infty
$$

Let $\mathcal{D}$ be the subspace of $L^{2}\left(\Gamma \backslash \mathbb{H}_{3}, d v\right) \cap C^{2}\left(\mathbb{H}_{3}\right)$ consisting of functions $f$ whose Laplacian $\Delta f$ is also square-integrable. It is known that $-\Delta$, regarded as an operator from $\mathcal{D}$ to $L^{2}\left(\Gamma \backslash \mathbb{H}_{3}\right)$, is essentially selfadjoint and positive [Elstrodt et al. 1987]. We denote the selfadjoint extension of this operator by $-\Delta$ as well.

For a general $\Gamma \subset$ Iso $\mathbb{H}_{3}$ we put $\Gamma^{+}:=\Gamma \cap \mathrm{Iso}^{+} \mathbb{H}_{3}$ and write $L^{2}(\Gamma):=L^{2}\left(\Gamma^{+} \backslash \mathbb{H}_{3}, d v\right)^{\Gamma}$ for the closed subspace consisting of classes of $\Gamma$-invariant functions. Restriction gives a positive selfadjoint operator $-\Delta$ on $L^{2}(\Gamma)$.

By an application of Stokes' Theorem we know that for $f \in L^{2}(\Gamma)$ the following two statements are equivalent [Elstrodt et al. 1987; $\geq 1996$ ]:

1. $f$ lies in the domain of $-\Delta$ and satisfies

$$
-\Delta f=\lambda f \quad \text { for some } \lambda .
$$

2. For all $g \in L^{2}(\Gamma)$ we have

$$
\begin{gathered}
\int_{\Omega} r^{2}\left(f_{x} g_{x}+f_{y} g_{y}+f_{r} g_{r}\right) d v=\lambda \int_{\Omega} f g d v, \\
\text { with } d v=\frac{d x d y d z}{r^{3}} .
\end{gathered}
$$

We shall use this equivalence to symmetrize the operator $-\Delta$, which is asymmetric in the original coordinates. This also shows that for our eigenvalue problem we could have also considered the Hilbert space of complex $L^{2}$-functions.

\subsection{The Spectrum of the Laplace Operator}

The spectrum spec $\Gamma$ of $-\Delta$ on $L^{2}(\Gamma)$ splits into its discrete and continuous parts:

$$
\operatorname{spec} \Gamma=\operatorname{dspec} \Gamma \cup \operatorname{cspec} \Gamma \text {. }
$$

The continuous spectrum $\operatorname{cspec} \Gamma$ consists of a certain number of repeated intervals:

$$
\operatorname{cspec} \Gamma=\bigcup_{i=1}^{h_{\Gamma}}[1, \infty),
$$

where $h_{\Gamma}$ is the number of cusps of $\Gamma$ [Elstrodt et al. 1985; Hejhal 1983; Maaß 1949a; Maaß 1949b; Venkov 1981].

The discrete spectrum dspec $\Gamma$ is a discrete subset of $[0, \infty)$ containing 0 with multiplicity 1 . We shall use the notation

$$
\operatorname{dspec} \Gamma=\left\{0<\lambda_{\Gamma}^{1} \leq \lambda_{\Gamma}^{2} \leq \cdots\right\},
$$

writing eigenvalues with their multiplicity.

\subsection{Asymptotics of Eigenfunctions}

We consider here eigenvalues $\lambda \in \operatorname{dspec} \Gamma$, with $\lambda>1$. An eigenfunction $f \in L^{2}(\Gamma)$ corresponding to $\lambda$ will be $C^{\infty}$ on $\mathbb{H}_{3}$ by elliptic regularity.

We suppose now, by possibly replacing $\Gamma$ with one of its conjugates, that $\infty$ is a cusp of $\Gamma$. This means that there is a lattice $\Lambda \subseteq \mathbb{C}$ with

$\Gamma_{\infty}^{\prime}:=\Gamma \cap\left\{\left(\begin{array}{ll}1 & w \\ 0 & 1\end{array}\right): w \in \mathbb{C}\right\}=\left\{\left(\begin{array}{ll}1 & \mu \\ 0 & 1\end{array}\right): \mu \in \Lambda\right\}$.

For $v, w \in \mathbb{C}$ we put $\langle v, w\rangle=\frac{1}{2}(\bar{v} w+\bar{w} v)$ and denote the lattice dual to $\Lambda$ by

$$
\Lambda^{0}:=\{w \in \mathbb{C}:\langle\mu, w\rangle \in \mathbb{Z} \text { for all } \mu \in \Lambda\} .
$$

From (2.1) and the fact that $\lambda>1$, we get a Fourier expansion for $f$ [Elstrodt et al. $\geq 1996$ ]. Namely, for $(w, r) \in \mathbb{H}_{3}$, we have

$$
f(w, r)=\sum_{\mu \in \Lambda^{0} \backslash\{0\}} a_{\mu} K_{s}(2 \pi|\mu| r) e^{2 \pi i\langle\mu, w\rangle}
$$

where $a_{\mu} \in \mathbb{R}, s^{2}=1-\lambda$, and $K_{s}$ is a Bessel function that has for $r \rightarrow \infty$ the asymptotic behavior

$$
\sqrt{r} K_{s}(2 \pi|\mu| r) \sim c e^{-2 \pi|\mu| r}
$$

(see [Abramowitz and Stegun 1965, §§ 4.2.27, 9.2.3, 9.2.4, 9.6.4; Мaaß 1949a; 1949b]). 
Since the series for the Fourier expansion (2.3) of $f$ converges uniformly to $f$, we find an exponential decay of $f$ as $r \rightarrow \infty$.

We shall consider in our examples only groups $\Gamma$ where $\lambda_{\Gamma}^{1}>1$ is already known.

For eigenvalues $\lambda \leq 1$ we get a Fourier expansion of the eigenfunctions similar to (2.3) with some additional terms that decay polynomially as $r \rightarrow \infty$.

\section{SOME DISCONTINUOUS GROUPS AND THEIR FUNDAMENTAL DOMAINS}

This section will describe some discontinuous subgroups $\Gamma \subset$ Iso $\mathbb{H}_{3}$ together with a fundamental domain that will always be of the form

$$
\Omega=\left\{(x, y, r):(x, y) \in \mathcal{P}, r \geq \sqrt{1-x^{2}-y^{2}}\right\}
$$

where $\mathcal{P}$ is a closed triangle or quadrangle contained in the unit disc.

In 3.1 we treat the case when $\Omega$ is (combinatorially) a tetrahedron. Later we shall discuss examples of arithmetic groups (Sections 3.2-3.5) and a nonarithmetic group (Section 3.6).

To fix the notation, let $d$ be a positive integer and $K=\mathbb{Z}(\sqrt{-d})$ the corresponding imaginary quadratic number field with ring of integers $\mathcal{O}$. We use our identification of $\mathrm{PSL}_{2}(\mathbb{C})$ with the group of orientation-preserving isometries of $\mathbb{H}_{3}$ to embed $\mathrm{PSL}_{2}(\mathcal{O})$ into Iso $\mathbb{H}_{3}$. Then $\mathrm{PSL}_{2}(\mathcal{O})$ acts discontinuously on $\mathbb{H}_{3}$ with a quotient space that is of finite volume but not compact. It turns out that $\mathrm{PSL}_{2}(\mathcal{O})$ has a unique maximal discontinuous extension in Iso $\mathbb{H}_{3}$ [Elstrodt et al. $\left.\geq 1996\right]$. This group is called the extended Bianchi group $\mathrm{EB}(\mathcal{O})$.

\subsection{Tesselating Tetrahedra}

We consider here geodesic tetrahedra $\Theta \subset \mathbb{H}_{3}$ that have one vertex at $\infty$ and three finite vertices. We further suppose that $\Theta$ tesselates $\mathbb{H}_{3}$, that is, $\mathbb{H}_{3}$ can be covered without interior overlap by congruent copies of $\Theta$. Up to isometry such a tetrahedron is determined by the angles between its boundary planes. Looking down from the vertex at infinity we have this situation:

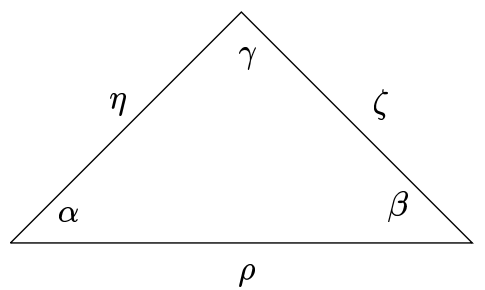

We can then represent the isometry types $\mathcal{T}$ by the symbol $\mathcal{T}=[\alpha, \beta, \gamma ; \eta, \zeta, \rho]$. An application of the Poincaré combination theorem shows that, up to isometry, there are only nine tetrahedra with the tesselating property [Elstrodt et al. $\geq 1996$ ]:

$$
\begin{aligned}
& \mathcal{T}_{1}=\left[45^{\circ}, 90^{\circ}, 45^{\circ} ; 90^{\circ}, 60^{\circ}, 90^{\circ}\right] \\
& \mathcal{T}_{2}=\left[90^{\circ}, 45^{\circ}, 45^{\circ} ; 90^{\circ}, 60^{\circ}, 90^{\circ}\right] \\
& \mathcal{T}_{3}=\left[30^{\circ}, 90^{\circ}, 60^{\circ} ; 90^{\circ}, 60^{\circ}, 90^{\circ}\right] \\
& \mathcal{T}_{4}=\left[60^{\circ}, 60^{\circ}, 60^{\circ} ; 90^{\circ}, 60^{\circ}, 90^{\circ}\right] \\
& \mathcal{T}_{5}=\left[30^{\circ}, 90^{\circ}, 60^{\circ} ; 90^{\circ}, 45^{\circ}, 90^{\circ}\right] \\
& \mathcal{T}_{6}=\left[60^{\circ}, 60^{\circ}, 60^{\circ} ; 90^{\circ}, 45^{\circ}, 90^{\circ}\right] \\
& \mathcal{T}_{7}=\left[30^{\circ}, 90^{\circ}, 60^{\circ} ; 90^{\circ}, 36^{\circ}, 90^{\circ}\right] \\
& \mathcal{T}_{8}=\left[60^{\circ}, 60^{\circ}, 60^{\circ} ; 90^{\circ}, 36^{\circ}, 90^{\circ}\right] \\
& \mathcal{T}_{9}=\left[45^{\circ}, 45^{\circ}, 90^{\circ} ; 60^{\circ}, 60^{\circ}, 90^{\circ}\right]
\end{aligned}
$$

Given a tetrahedron $\Theta$ from one of these classes, we may form the tesselation group $G(\Theta)$ of $\Theta$, that is, the subgroup of Iso $\mathbb{H}_{3}$ generated by the reflections in the boundary planes of $\Theta$. It is known that $G(\Theta)$ acts discontinuously on $\mathbb{H}_{3}$ and has $\Theta$ as fundamental domain. Since $\Theta$ also has finite hyperbolic volume, $G(\Theta)$ is one of the groups discussed in the Introduction.

Take $\Theta \in \mathcal{T}_{1}$. By doubling $\Theta$ through a suitable boundary plane we obtain a tetrahedron $\Theta^{\prime} \in \mathcal{T}_{2}$. It is then easy to see that $G(\Theta)$ is of index two over $G\left(\Theta^{\prime}\right)$. The same situation also holds for the pairs $\left(\mathcal{T}_{3}, \mathcal{T}_{4}\right),\left(\mathcal{T}_{5}, \mathcal{T}_{6}\right),\left(\mathcal{T}_{7}, \mathcal{T}_{8}\right)$.

The groups for the tetrahedra from $\mathcal{T}_{1}, \mathcal{T}_{3}, \mathcal{T}_{5}, \mathcal{T}_{7}$ will appear below. The group corresponding to a tetrahedron from $\mathcal{T}_{9}$ is conjugate to a group commensurable with $\mathrm{PSL}_{2}(\mathbb{Z}[i])$ and will not be discussed further. 


\section{2. $\mathrm{PSL}_{2}(\mathbb{Z}[i])$}

A fundamental domain for $\mathrm{PSL}_{2}(\mathbb{Z}[i])$, first given in [Picard 1884], is described as follows:

$$
\begin{aligned}
\Upsilon_{1}=\{ & (x, y, r): \\
& \left.-\frac{1}{2} \leq x \leq \frac{1}{2}, 0 \leq y \leq \frac{1}{2}, r \geq \sqrt{1-x^{2}-y^{2}}\right\}
\end{aligned}
$$

[Elstrodt et al. 1987; Stramm 1994; Swan 1971]. $\Upsilon_{1}$ is a hyperbolic pyramid with top point at $\infty$ and all other points finite.

The boundary identifications of $\Upsilon_{1}$ by $\mathrm{PSL}_{2}(\mathbb{Z}[i])$

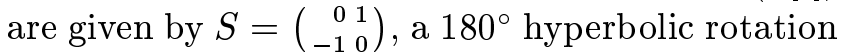
around the line with endpoints $(z, r)=( \pm i, 0)$; $T_{1}=\left(\begin{array}{ll}1 & 1 \\ 0 & 1\end{array}\right)$, a parabolic transformation mapping $(z, r)$ to $(z+1, r) ; T_{i}=\left(\begin{array}{ll}1 & i \\ 0 & 1\end{array}\right)$, a parabolic transformation mapping $(z, r)$ to $(z+i, r)$; and $U_{\pi}=$ $\left(\begin{array}{cc}i & 0 \\ 0 & -i\end{array}\right)$, a $180^{\circ}$ rotation around the $r$-axis.

From the identification $S$ we get for a $\Gamma$-invariant Laplace eigenfunction $f$ the condition

$$
f(x, y, r)=f(-x, y, r)
$$

on the bottom boundary of $\Upsilon_{1}$ (a piece of the hyperbolic plane defined by $\left.x^{2}+y^{2}+r^{2}=1\right)$. From the identifications $T_{1}, T_{1}^{-1}, U_{\pi}$, and $T_{i} U_{\pi}$ among other pieces of the boundary of $\Upsilon_{1}$ we get the additional conditions

$$
\begin{aligned}
& f\left(x, \frac{1}{2}, r\right)=f\left(-x, \frac{1}{2}, r\right), \\
& f\left(\frac{1}{2}, y, r\right)=f\left(-\frac{1}{2}, y, r\right), \\
& f(x, 0, r)=f(-x, 0, r) .
\end{aligned}
$$

The extended Bianchi group $\operatorname{EB}(\mathbb{Z}[i])$, which is of index four over $\mathrm{PSL}_{2}(\mathbb{Z}[i])$, has

$$
\begin{aligned}
\Theta_{1}=\{(x, y, r): & \\
& \left.0 \leq x \leq \frac{1}{2}, 0 \leq y \leq x, r \geq \sqrt{1-x^{2}-y^{2}}\right\}
\end{aligned}
$$

as fundamental domain [Bianchi 1892].

The tetrahedron $\Theta_{1}$ is in the class $\mathcal{T}_{1}$ and has volume approximately $0.0763 . \mathrm{EB}(\mathbb{Z}[i])$ is the tesselation group of $\Theta_{1}$. Concerning the eigenvalues of the Laplace operator we know [Elstrodt et al. 1987; Stramm 1994] that

$$
\lambda_{\mathrm{PSL}_{2}(\mathbb{Z}[i])}^{1} \geq \frac{2}{3} \pi^{2} \approx 6.58 .
$$

\section{3. $\mathrm{PSL}_{2}(\mathbb{Z}[\sqrt{-2}])$}

Put $\xi:=\sqrt{-2}$. Then $\mathbb{Z}[\xi]$ is the ring of integers in the number field $\mathbb{Z}(\sqrt{-2})$. A fundamental domain of $\mathrm{PSL}_{2}(\mathbb{Z}[\xi])$ is

$$
\begin{aligned}
& \Upsilon_{2}=\{(x, y, r): \\
& \left.\quad-\frac{1}{2} \leq x \leq \frac{1}{2},-\frac{\sqrt{2}}{2} \leq y \leq \frac{\sqrt{2}}{2}, r \geq \sqrt{1-x^{2}-y^{2}}\right\} .
\end{aligned}
$$

The boundary identifications come from $S, T_{1}$, the parabolic $T_{\xi}=\left(\begin{array}{ll}1 & \xi \\ 0 & 1\end{array}\right), T_{1}^{-1}$ and $T_{\xi}^{-1}$. A Laplace eigenfunction $f$ satisfies

$$
f(x, y, r)=f(-x, y, r)
$$

on the bottom boundary of $\Upsilon_{2}$. On the vertical boundary pieces we get

$$
\begin{aligned}
f\left(x, \frac{\sqrt{2}}{2}, r\right) & =f\left(x,-\frac{\sqrt{2}}{2}, r\right), \\
f\left(\frac{1}{2}, y, r\right) & =f\left(\frac{1}{2},-y, r\right) .
\end{aligned}
$$

A fundamental domain of the extended Bianchi group $\operatorname{EB}(\mathbb{Z}[\xi])$, which is again of index four over $\mathrm{PSL}_{2}(\mathbb{Z}[\xi])$, is

$$
\begin{aligned}
\Theta_{2}=\{ & (x, y, r): \\
& \left.0 \leq x \leq \frac{1}{2}, 0 \leq y \leq \frac{\sqrt{2}}{2}, r \geq \sqrt{1-x^{2}-y^{2}}\right\} .
\end{aligned}
$$

The volume of $\Theta_{2}$ is approximately 0.2509 . It is known that

$$
\lambda_{\mathrm{PSL}_{2}(\mathbb{Z}[\xi])}^{1} \geq \frac{1}{4} \pi^{2} \approx 2.47
$$

[Elstrodt et al. 1987; Stramm 1994].

\section{4. $\mathrm{PSL}_{2}\left(\mathbb{Z}\left[\frac{1}{2}(-1+\sqrt{-3})\right]\right)$}

Let $\omega$ be the cube root of unity $\frac{1}{2}(-1+\sqrt{-3})$. Then $\mathbb{Z}[\omega]$ is the ring of integers in $\mathbb{Z}(\sqrt{-3})$. A fundamental domain of $\mathrm{PSL}_{2}(\mathbb{Z}[\omega])$ is

$$
\begin{aligned}
\Upsilon_{3}=\{ & (x, y, r): 0 \leq x \leq \frac{1}{2}, \\
& \left.-\frac{1}{\sqrt{3}} x \leq y \leq \frac{1}{\sqrt{3}}(1-x), r \geq \sqrt{1-x^{2}-y^{2}}\right\}
\end{aligned}
$$

[Stramm 1994; Swan 1971]. The boundary identifications are given by $T_{\omega}=\left(\begin{array}{ll}1 & \omega \\ 0 & 1\end{array}\right)$, a parabolic transformation taking $(z, t)$ to $(z+\omega, t)$;

$$
U_{2 \pi / 3}=\left(\begin{array}{cc}
e^{-i \pi / 3} & 0 \\
0 & e^{i \pi / 3}
\end{array}\right),
$$


a $120^{\circ}$ rotation around the $r$-axis; and $S, T_{1}, U_{2 \pi / 3}$, $U_{2 \pi / 3}^{-1}, U_{2 \pi / 3}^{-1} S, U_{2 \pi / 3}^{-1} T_{1}, U_{2 \pi / 3} T_{1} T_{\omega}$.

The extended Bianchi group $\operatorname{EB}(\mathbb{Z}[\omega])$ is of index four over $\mathrm{PSL}_{2}(\mathbb{Z}[\omega])$ and has

$$
\begin{aligned}
\Theta_{3}= & \{(x, y, r): \\
& \left.0 \leq x \leq \frac{1}{2}, 0 \leq y \leq \frac{1}{\sqrt{3}} x, r \geq \sqrt{1-x^{2}-y^{2}}\right\}
\end{aligned}
$$

as fundamental domain [Bianchi 1892]. $\Theta_{3}$ is in the class $\mathcal{T}_{3}$ and has volume approximately 0.0422 . $\operatorname{EB}(\mathbb{Z}[\omega])$ is the group of the tesselation with $\Theta_{3}$. It is known that

$$
\lambda_{\mathrm{PSL}_{2}(\mathbb{Z}[\omega])}^{1} \geq \frac{32}{27} \pi^{2} \approx 11.70
$$

[Elstrodt et al. 1987; Stramm 1994].

\section{5. $E_{4}$ and $\Gamma_{4}$}

Here we start off with the tetrahedron

$$
\begin{aligned}
\Theta_{4} & =\{(x, y, r): \\
& \left.0 \leq x \leq \frac{1}{\sqrt{2}}, 0 \leq y \leq \frac{1}{\sqrt{3}} x, r \geq \sqrt{1-x^{2}-y^{2}}\right\},
\end{aligned}
$$

which is in the class $\mathcal{T}_{5}$. We define $E_{4}$ to be the group of the tesselation given by $\Theta_{4}$. By looking at the angles of $\Theta_{4}$ one can see that $E_{4}$ contains a subgroup $\Gamma_{4} \subset \mathrm{PSL}_{2}(\mathbb{C})$ of index four, which has the pyramid

$$
\begin{aligned}
\Upsilon_{4}=\{ & (x, y, r): 0 \leq x \leq \frac{1}{\sqrt{2}}, \\
& \left.-\frac{1}{\sqrt{3}} x \leq y \leq \frac{1}{\sqrt{3}}(1-x), r \geq \sqrt{1-x^{2}-y^{2}}\right\}
\end{aligned}
$$

as fundamental domain. The group $\Gamma_{4}$ is commensurable with $\mathrm{PSL}_{2}(\mathbb{Z}[\omega])$, with commensurability index $\frac{5}{2}$. The volume of $\Theta_{4}$ is approximately 0.1056 . It can be proved, using the method described in [Elstrodt et al. 1987; Stramm 1994], that

$$
\lambda_{\Gamma_{4}}^{1}>1
$$

3.6. $E_{5}$ and $\Gamma_{5}$

Here we start off with the tetrahedron

$$
\begin{aligned}
\Theta_{5}=\{(x, y, r): 0 & \leq x \leq \frac{1}{4}(1+\sqrt{5}), \\
0 & \left.\leq y \leq \frac{1}{\sqrt{3}} x, r \geq \sqrt{1-x^{2}-y^{2}}\right\},
\end{aligned}
$$

which is in the class $\mathcal{T}_{7}$. We define $E_{5}$ to be the group of the tesselation given by $\Theta_{5}$. By looking at the angles of $\Theta_{5}$ it can be seen that $E_{5}$ contains a subgroup

$$
\Gamma_{5} \subset \mathrm{PSL}_{2}(\mathbb{C})
$$

of index four, which has the pyramid

$$
\begin{aligned}
\Upsilon_{5}=\{ & (x, y, r): 0 \leq x \leq \frac{1}{4}(1+\sqrt{5}) \\
& \left.-\frac{1}{\sqrt{3}} x \leq y \leq \frac{1}{\sqrt{3}}(1-x), r \geq \sqrt{1-x^{2}-y^{2}}\right\}
\end{aligned}
$$

as fundamental domain.

By the arithmeticity criterion of Vinberg, $E_{5}$ is not arithmetic. A look at its fundamental domain shows that $E_{5}$ is maximal discontinuous. By an arithmeticity criterion of Margulis $E_{5}$ is also equal to its commensurator; see [Elstrodt et al. $\geq 1996$ ] for more details.

The volume of $\Theta_{5}$ is approximately 0.1732 . By the method of [Elstrodt et al. 1987; Stramm 1994] we can prove that

$$
\lambda_{\Gamma_{5}}^{1}>1
$$

The boundary identifications for $\Upsilon_{4}$ and $\Upsilon_{5}$ are analogous to those of $\Upsilon_{3}$, and induce similar symmetries.

\subsection{A Family of Tetrahedra}

For later use we introduce a continuous family of tetrahedra. If $x_{Q}$ and $y_{Q}$ are positive reals with $x_{Q}<1$ and $y_{Q}^{2}<1-x_{Q}^{2}$, we set

$$
\begin{aligned}
\Theta\left(x_{Q}, y_{Q}\right):= & \left\{(x, y, r): 0 \leq x \leq x_{Q},\right. \\
& \left.0 \leq y \leq x y_{Q} / x_{Q}, \sqrt{1-x^{2}-y^{2}} \leq r\right\}
\end{aligned}
$$

For suitable values of the parameters, $\Theta\left(x_{Q}, y_{Q}\right)$ coincides with the tesselating tetrahedra discussed before.

One edge of $\Theta\left(x_{Q}, y_{Q}\right)$ always passes through the north pole of the unit sphere. The angle between the vertical boundary planes meeting there is called northern angle. 


\section{DISCRETIZATION}

We describe here the general principles we use to find appropriate solutions of the differential equation

$$
-\Delta f=\lambda f
$$

\subsection{Cut-off}

Consider the equation (4.1) on polyhedra $\Omega \subseteq \mathbb{H}_{3}$ of type (3.1) with boundary $\partial \Omega$. We want to find solutions that satisfy certain compatibility conditions on $\partial \Omega$ and that have a rapid decay for $r \rightarrow \infty$. So it seems reasonable to replace the unbounded polyhedron

$$
\Omega=\{(x, y, r):(x, y) \in \mathcal{P}, r>0\}
$$

by the bounded region

$$
\Omega_{R}=\{(x, y, r) \in \Omega: r \leq R\},
$$

where $R$ is supposed to be reasonably big. The new regions $\Omega_{R}$ have an additional upper boundary, which we call $\partial \Omega_{0}$.

We now search for solutions of our differential equation (4.1) on the regions $\Omega_{R}$. To recognize amongst the solutions obtained the desired eigenfunctions, we consider the following two additional boundary conditions:

1. the Dirichlet condition $u=0$ on $\partial \Omega_{0}$, and

2. the Neumann condition $\partial u / \partial n=0$ on $\partial \Omega_{0}$.

We look for solutions of (4.1) on $\Omega_{R}$ separately under the Dirichlet boundary condition and under the Neumann boundary condition. If two such solutions coincide, taking into account the quality of the approximation, both come from a rapidly decaying solution on $\Omega$.

In each case, we usually start by applying our procedure with $R=15$, and then again with $R=$ 19 , in order to compare the results. If the differences in the eigenvalue approximations were considerably smaller than the error predicted by the choice of the actual finite elements [Axelsson and
Barker 1984; Zienkiewicz and Morgan 1983] we accepted the chosen cut-off height; otherwise we increased it.

\subsection{Division of Polyhedra}

Suppose our polyhedron $\Omega$ admits a symmetry by a hyperbolic reflection $\sigma$ in some vertical plane that cuts $\Omega$ into to congruent pieces $\Omega^{\prime}$ and $\Omega^{\prime \prime}$. We write $\partial \Omega_{1}$ for the common boundary of $\Omega^{\prime}$ and $\Omega^{\prime \prime}$.

Since the space of solutions for given $\lambda$ decomposes into an even and an odd part [Huntebrinker 1995; Smotrov and Golovchansky 1991], it is sometimes convenient to consider our problem only on one half, say $\Omega^{\prime}$, of $\Omega$. Since the plane of symmetry is vertical we may then use the cut-off regions $\Omega_{R}^{\prime}$.

To get the even eigenfunctions we look for solutions on $\Omega_{R}^{\prime}$ of (4.1) subject to the boundary conditions

$$
u=0 \quad \text { on } \partial \Omega_{0}, \quad \frac{\partial u}{\partial n}=0 \quad \text { on } \partial \Omega_{1},
$$

and then for solutions subject to the boundary conditions

$$
\frac{\partial u}{\partial n}=0 \quad \text { on } \quad \partial \Omega_{0} \cup \partial \Omega_{1} .
$$

The desired eigenfunctions of rapid decay are those that are solutions for both sets of boundary conditions.

To get the odd eigenfunctions we impose the boundary condition

$$
u=0 \quad \text { on } \partial \Omega_{0} \cup \partial \Omega_{1} .
$$

The numerical computations indicate that all solutions of (4.1) under this boundary condition have the required rapid decay.

\subsection{Weak Formulation}

We want to apply the finite element method to our eigenvalue problem. To do this we need a weak formulation of the problem [Babuška and Osborn 1991; Chatellin 1983; Hackbusch 1986].

We use the equivalence of the differential equation (2.1) and the integral equation (2.2) and get the following weak formulation: 
Determine $u \in V$ such that, for all $v \in V$,

$$
\begin{array}{r}
\iiint_{\Omega_{R}} \frac{1}{r}\left(u_{x} v_{x}+u_{y} v_{y}+u_{r} v_{r}\right) d x d y d r= \\
\lambda \iiint_{\Omega_{R}} \frac{1}{r^{3}} u v d x d y d r,
\end{array}
$$

where $V$ is the Sobolev space $H_{0}^{1}\left(\Omega_{R}\right)$ if there is a Dirichlet boundary condition, or the Sobolev space $H^{1}\left(\Omega_{R}\right)$ if all boundary conditions are Neumann.

Note that the weak formulation gives a symmetric eigenvalue problem with real eigenvalues and eigenfunctions.

\section{NUMERICAL METHODS}

In this section we discuss the actual algorithms used, and comment on our methods of triangulation.

The reader unfamiliar with finite element methods can turn to the books [Axelsson and Barker 1984; Hughes 1987; Schwarz 1991; Zienkiewicz and Morgan 1983] for general information.

\subsection{The Problem}

We consider our cut-off region $\Omega_{R} \subset \mathbb{R}^{2} \times \mathbb{R}_{+}$, as described in the previous section. Let $\partial \Omega_{R}$ be the boundary of $\Omega_{R}$ and $\partial \Omega_{0}$ the top boundary. We suppose, as indicated in the last section, that the remaining boundary is decomposed into parts $\partial \Omega_{i}$, $i=1, \ldots, j$. We wish to numerically solve the eigenvalue problem (4.4), subject to $j+1$ boundary conditions of the form (4.2) or (4.3), and the additional condition

$$
\iiint_{\Omega_{R}} \frac{1}{r^{3}} u d x d y d r=0
$$

If the $j+1$ boundary conditions are all of Dirichlet type on $\partial \Omega_{R}$, the side condition (5.1) is optional. Otherwise it is necessary in order to make the eigenvalue problem uniquely solvable [Hackbusch 1986].
To sum up, a choice of a triangulation will lead to a matrix equation of the following type:

$$
\left(\begin{array}{ll}
\boldsymbol{u}^{T} & c
\end{array}\right)\left(\begin{array}{cc}
\boldsymbol{A} & \boldsymbol{h} \\
\boldsymbol{h}^{T} & 0
\end{array}\right)\left(\begin{array}{c}
\boldsymbol{u} \\
c
\end{array}\right)=\lambda\left(\begin{array}{c}
\boldsymbol{u}^{T} \boldsymbol{B} \boldsymbol{u} \\
0
\end{array}\right)
$$

\subsection{Decomposition into Standard Regions}

As described in the previous section, the total region $\Omega_{R}$ is decomposed using additional symmetries into a certain number of congruent standard regions. The standard regions we encounter are truncated hyperbolic pyramids, given for suitable $x_{Q}$ and $y_{Q}$ by the equations

$$
\begin{aligned}
\Phi\left(x_{Q}, y_{Q}\right)_{R}= & \left\{(x, y, r): 0 \leq x \leq x_{Q},\right. \\
-x y_{Q} \leq & \left.y x_{Q} \leq x y_{Q}, \sqrt{1-x^{2}-y^{2}} \leq r \leq R\right\} \\
\Psi\left(x_{Q}, y_{Q}\right)_{R}= & \left\{(x, y, r): 0 \leq x \leq x_{Q},\right. \\
& \left.0 \leq y \leq y_{Q}, \sqrt{1-x^{2}-y^{2}} \leq r \leq R\right\} .
\end{aligned}
$$

We refer to these regions as triangular or rectangular prisms, respectively, because of their appearance in upper half-space (Figure 1). These prisms are bounded below by the unit sphere.

Figure 1 also shows our notation for the edges. Edge $\mathrm{N}$ always passes through the north pole of the unit sphere. The angle between planes NP and NQ is called the northern angle. The prism $\Phi\left(x_{Q}, y_{Q}\right)_{R}$ is a cut-off version of double the tetrahedron $\Theta\left(x_{Q}, y_{Q}\right)$ introduced in Section 3 .

The stiffness matrix $\boldsymbol{A}$ and the mass matrix $\boldsymbol{B}$ in (5.2) are only computed for one standard region. The total matrices are not stored; they only appear implicitly when boundary conditions are introduced [Huntebrinker 1995]. This procedure leads to a considerable amount of saving of storage space, and makes the computation feasible on smaller machines. A disadvantage is the bad vectorizability of matrix multiplication, but on the IBM RS/6000 used by us this played no role.

The Dirichlet boundary conditions are enforced by the elimination of all nodes from our eigenvalue problem [Schwarz 1991; Huntebrinker 1995]. In 


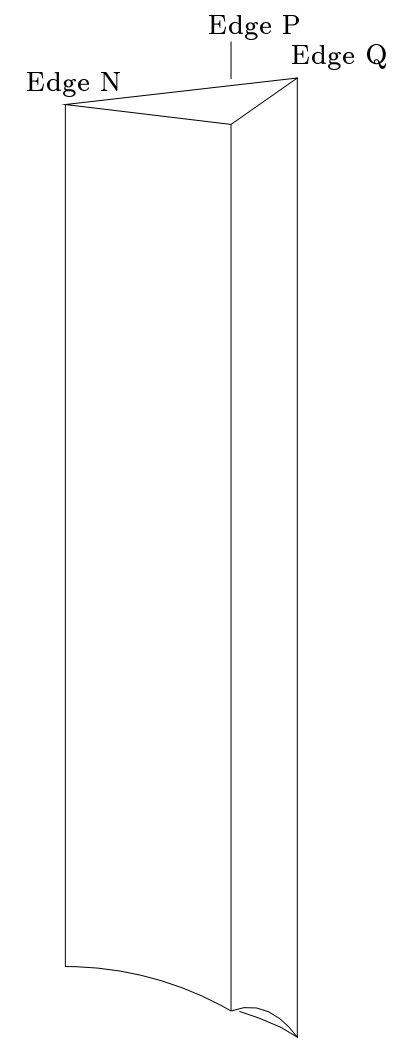

Truncation of $\Theta\left(x_{Q}, y_{Q}\right)$
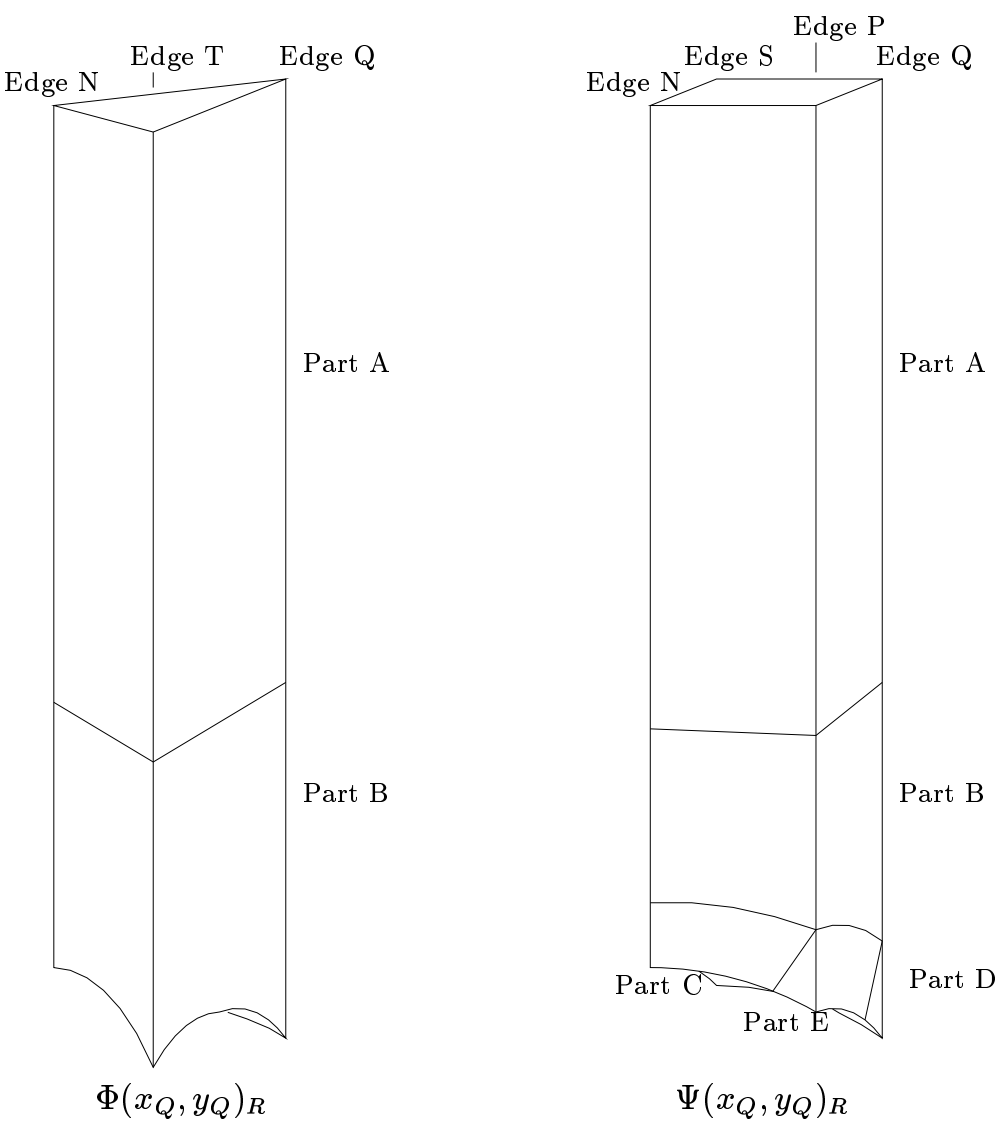

FIGURE 1. Decomposition of the standard regions, showing also the labeling of vertical edges. Parts A-E correspond to different methods of subdivision into elements (Section 5.5).

this way the size of the system of linear equations is reduced by the number of Dirichlet boundary points.

\subsection{Choice of Elements}

Since the desired eigenfunctions are sufficiently regular, it is possible to use higher-degree elements [Hackbusch 1986; Babuška and Guo 1988; Babuška et al. 1989; Babuška and Osborn 1991]. Quadratic or cubic elements are particularly suited to eigenvalue problems because they reduce the size of the final system of linear equations. The biggest gain occurs by using elements from the serendipity family without inner nodes [Ergatoudis et al. 1968; Hughes 1987; Schwarz 1991; Zienkiewicz and Morgan 1983]; see also [Huntebrinker 1991; Huntebrinker 1995].
For the approximation of the sphere caps at the bottom we have to use isoparametric elements. The quality of the approximation here depends on the choice of the nodes on the curved boundary [Axelsson and Barker 1984; Banerjee 1992; Banerjee and Osborn 1990; Ciarlet 1978; Ciarlet and Raviart 1972; Schwarz 1991; Zienkiewicz and Morgan 1983].

It is especially important to avoid obtuse inner angles in the elements. Acute angles give significantly better approximations [Babuška and Aziz 1976; Jamet 1976; Krizek 1992]. In order for only acute angles to occur we use tetrahedral elements near the points where the vertical planes meet the sphere.

In the areas of big $r$ we use parallelepiped elements, which offer the possibility of a finer adjustment of the triangulation. 


\subsection{Adaptive Refinements}

By adaptive refinement we mean the refinement of a triangulation with the aim of minimizing the global error in the computation of an iterated function, by minimizing local errors. See [Babuška 1986; 1988; Babuška and Guo 1992; Ewing 1990; Strouboulis and Haque 1992; Zienkiewicz and Zhu 1991] for a discussion of standard methods.

To get good indicators for the local errors of the iterated functions we use a method that we will describe below. Our choice of refinement leads to restricted adaptivity [Huntebrinker 1995].

Taking the strong decay in $r$-direction of our desired solutions into consideration we use in the lower parts of the standard region a global strategy of refinement. In the upper parts we use an adaptive refinement with the size of the iterated function as error indicator. If the cut-off height is chosen big enough this leads to reasonable results. For our actual choice of cut-off heights, see the explanation in Section 4.1.

For the computation of the error indicators it is important to have no eigenfunctions without strong decay present, because such eigenfunctions force an excessive refinement in the upper areas. We therefore choose the functions for the computation of the indicator after some analysis of their growth.

\subsection{Triangulation of the Standard Regions}

We now describe how our standard regions (prisms) are triangulated. Rectangular prisms are cut into five areas (see Figure 1, right):

1. In area $A$, which is above some horizontal plane, we use parallelepiped elements. The refinement is controlled by the error indicator described above.

2. Just below area $\mathrm{A}$ we have area $B$, which is bounded above by a horizontal plane and below by a surface that models the surface of the sphere. Here we use parallelepipeds that get shorter when approaching the $r$-axis.

3. Between the curved surface and the sphere cap at the bottom we get area $C$, where we make the parallelepiped elements shorter as we move in the direction of the $x r$ - and $y r$-planes. This cuts off two wedges along the lower left-hand edge and along the rear edge.

4. Between the wedges, the surface of the sphere and the boundary planes area $D$, which is a pyramid, appears. This area is decomposed using tetrahedral elements.

5. The union of the two wedges, area $E$, is decomposed into prism elements with triangles as cross-sections.

Triangular prisms have a simpler triangulation, as shown in Figure 1, middle: in area $A$ with a refinement controlled by an error indicator, and in area $B$ with a global refinement strategy, using a variation of methods of Bank and Sherman for computing the subsequent triangulations [Bank 1983; Bank and Sherman 1979; 1981; Bank et al. 1983].

\subsection{Iteration Methods and Analysis of Eigenfunctions}

We use simultaneous vector iteration together with the method of conjugate gradients. For these procedures see [Concus et al. 1976; Hackbusch 1993; Hestenes and Stiefel 1952; Schwarz 1991; Babuška and Osborn 1987; 1989; 1991]. The eigenvalues are determined here by computation of a Rayleigh quotient. See [Sartoletto et al. 1989; Gambolati and Putti 1994; Watkins 1993] for an overview of methods for the computation of eigenvalues of big matrices. For the details of our procedure we refer to [Huntebrinker 1995]. For the iteration method used it is important not to have too much need of storage space.

To judge the decay of the appearing functions we compute maximal and minimal values in the upper areas of our region. These data also offer the possibility of seeing symmetries of the function.

\section{SYMMETRIES OF EIGENFUNCTIONS}

There are two distinct situations:

1. If $\Theta_{i}$ is a tetrahedron, so its truncation is a rectangular prism, we consider the union of $\Theta_{i}$ with 

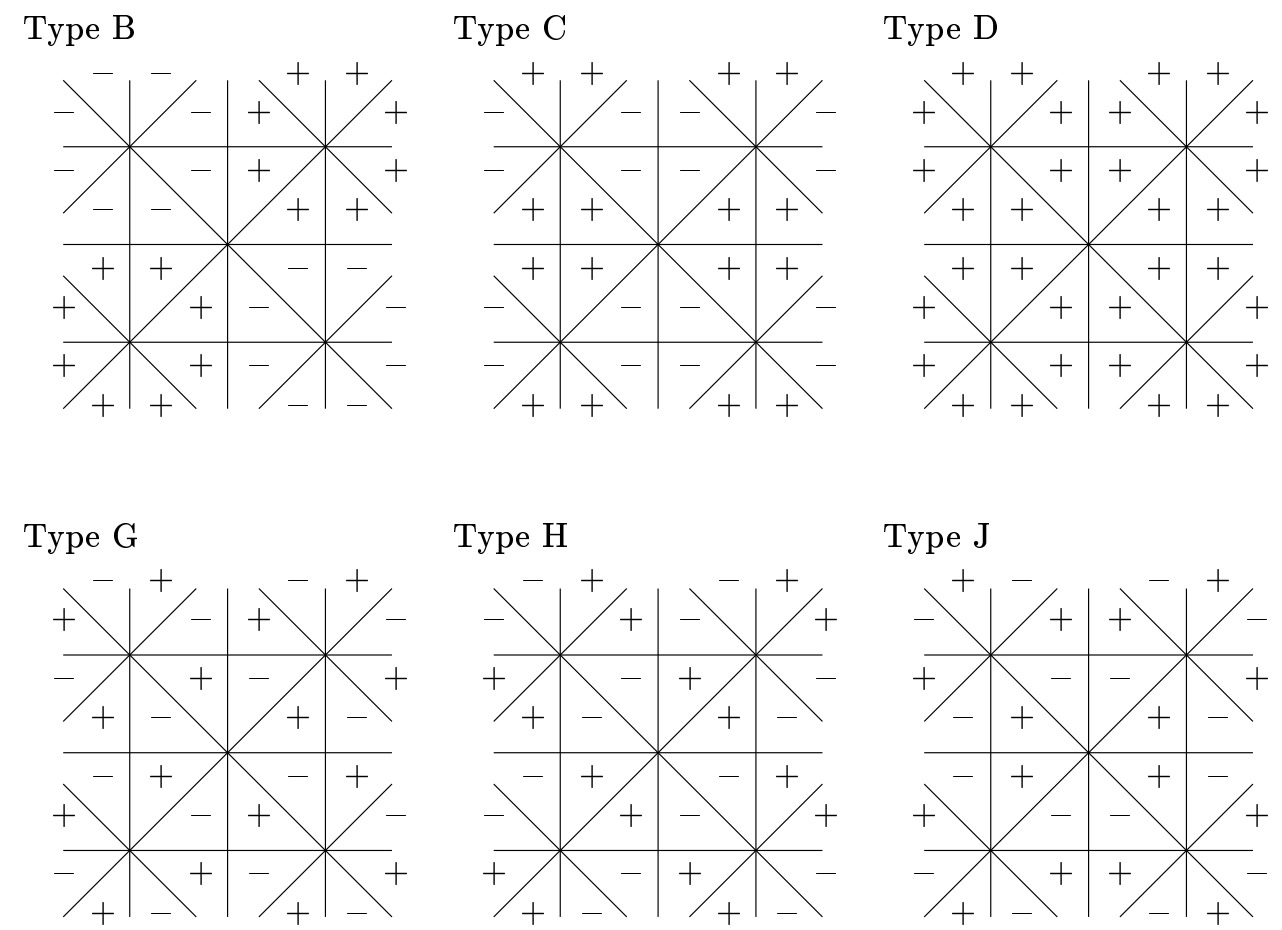

FIGURE 2. Symmetries of eigenfunctions for a triangular prism with a northern angle of $45^{\circ}$.

its reflection in the plane NQ. The newly arising vertical edge is called $\mathrm{T}$. The eigenspace with respect to a given $\lambda$ decomposes into subspaces of functions that are either even or odd with respect to each of the boundary planes (the bottom, NT, NQ, and QT).

2. If $\Theta_{i}$ is a pyramid, so its truncation is a rectangular prism, the eigenspace with respect to a given $\lambda$ decomposes into subspaces of functions that are either even or odd with respect to the boundary planes (the bottom, NP, PQ, QS, and NS).

Of course, not all combinations of symmetries are possible. We now describe and name the cases that do arise.

\subsection{Symmetries for a Triangular Prism}

For $\Upsilon_{1}, \Upsilon_{3}, \Upsilon_{4}$, and $\Upsilon_{5}$, six types of symmetries can arise, as listed below. The edge $\mathrm{P}$ is the same as in the explanation above. See also Figures 2 and 3 .
Type B: even with respect to the bottom, NQ, and PQ; odd with respect to NP.

Type C: even with respect to the bottom, NP, and

PQ; odd with respect to NQ.

Type D: even with respect to all boundary planes. Type G: odd with respect to all boundary planes.

Type H: odd with respect to the bottom, NP, and

PQ; even with respect to NQ.

Type J: odd with respect to the bottom, NQ, and

PQ; even with respect to NP.

On $\Upsilon_{1}$, the fundamental region of $\mathrm{PSL}_{2}(\mathbb{Z}[i])$, we have eigenfunctions of types $\mathrm{C}, \mathrm{D}, \mathrm{G}$ and $\mathrm{H}$ (see Figure 2). On $\Upsilon_{3}, \Upsilon_{4}$ and $\Upsilon_{5}$, we have the types $\mathrm{B}, \mathrm{D}, \mathrm{G}$ and $\mathrm{J}$ (see Figure 3 ).

On the fundamental regions $\Theta_{1}, \Theta_{3}, \Theta_{4}$ and $\Theta_{5}$ we only have eigenfunctions of type $\mathrm{D}$.

\subsection{Symmetries for a Rectangular Prism}

In the computations on $\Upsilon_{2}$ we get eigenfunctions of four types of symmetry. For the polyhedron in 

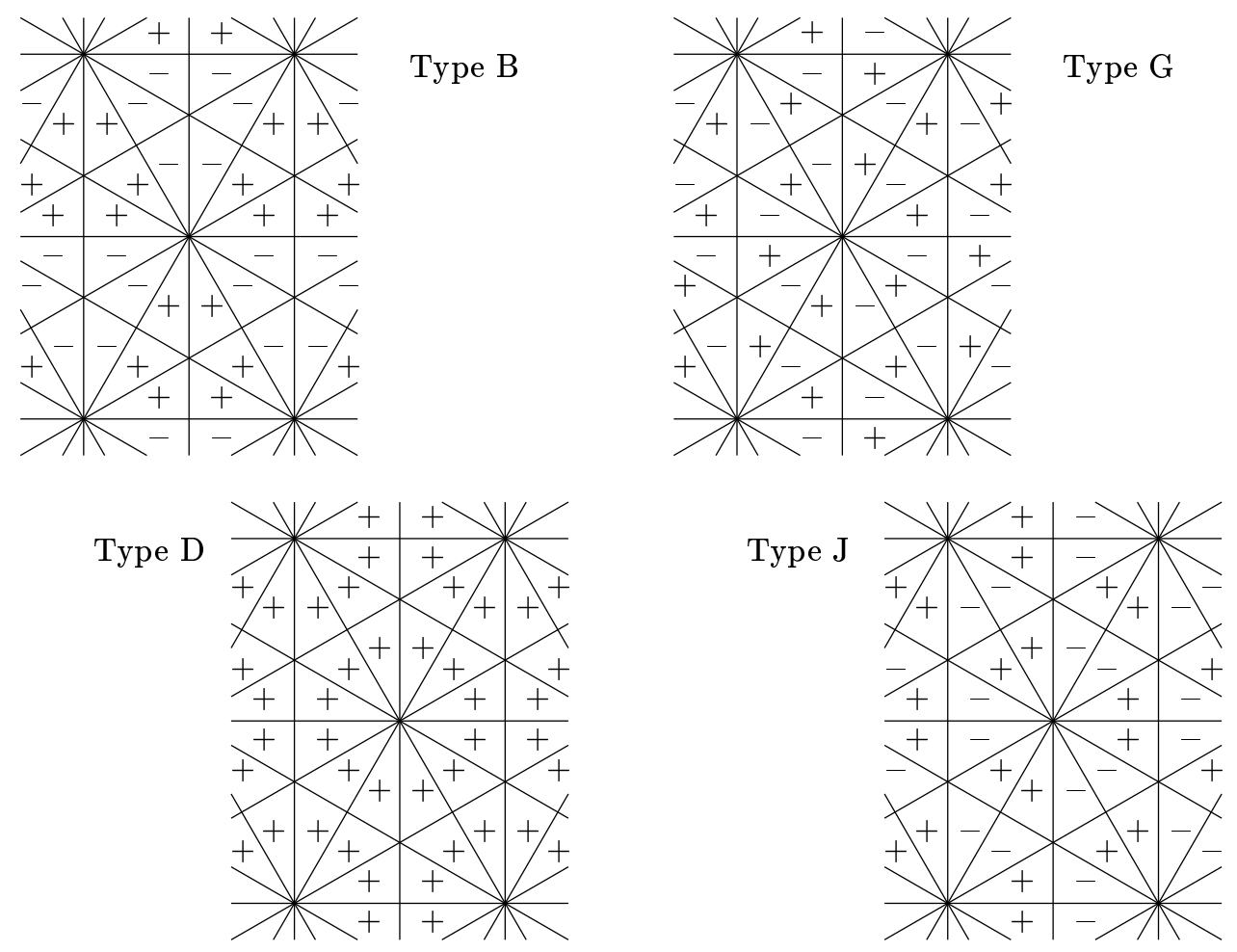

FIGURE 3. Symmetries of eigenfunctions for a triangular prism with a northern angle of $30^{\circ}$.

Type D

\begin{tabular}{c|c|c|c}
+ & + & + & + \\
\hline+ & + & + & + \\
\hline+ & + & + & + \\
\hline+ & + & + & +
\end{tabular}

Type $\mathrm{H}$

\begin{tabular}{c|c|c|c}
- & + & - & + \\
\hline+ & - & + & - \\
\hline- & + & - & + \\
\hline+ & - & + & -
\end{tabular}

Type $\mathrm{P}$

\begin{tabular}{c|c|c|c}
+ & - & + & - \\
\hline+ & - & + & - \\
\hline+ & - & + & - \\
\hline+ & - & + & -
\end{tabular}

Type T

\begin{tabular}{c|c|c|c}
- & - & - & - \\
\hline+ & + & + & + \\
\hline- & - & - & - \\
\hline+ & + & + & +
\end{tabular}

FIGURE 4. Symmetries of eigenfunctions for a rectangular prism. 
Figure 1, with edges N, P, Q and S, they are as follows:

Type D: even with respect to all boundary planes. Type H: odd with respect to all boundary planes. Type P: even with respect to the bottom, NP, and QS; odd with respect to PQ and NS.

Type T: odd with respect to the bottom, NP, and

QS; even with respect to PQ and NS.

See Figure 4. On $\Theta_{2}$ we only have eigenfunctions of type D.

\section{NUMERICAL COMPUTATIONS}

\subsection{Computation for the Fundamental Regions}

For $\Upsilon_{1}$ we get the best approximations using cubic elements. We end up with systems of linear equations of degree roughly 30000 for type C, 7000 for type D, 9800 for type G, and 5700 for type H. Computations using quadratic elements are less precise but have confirmed our results.

For $\Upsilon_{2}$ we also used cubic elements. We got systems of linear equations of size 6000 for type D, 3700 for type H, 9400 for type $\mathrm{P}$, and 7800 for type $\mathrm{T}$.

For $\Upsilon_{3}$ we used quadratic elements and got systems of equations of sizes between 10000 and 12000 .

For $\Upsilon_{4}$ and $\Upsilon_{5}$ we had to use finer meshes; the orders of our systems of equations were 30000 to 35000 .

\subsection{Computation for the Deformed Fundamental Regions}

This reports on our computations for the family of tetrahedra $\Theta\left(x_{Q}, y_{Q}\right)$ introduced in Section 3. We have studied the variation of eigenvalues for types $\mathrm{B}, \mathrm{C}$ and D. For the corresponding eigenfunctions $f$ this means that, apart from exponential decay, the following conditions must be satisfied:

Type B: the Neumann condition on the bottom and on the planes NQ and PQ, and the Dirichlet condition on NP.
Type C: the Neumann condition on the bottom and on the planes NP and PQ, and the Dirichlet condition on NQ.

Type D: the Neumann condition on all the boundary.

We have carried out three series of computations:

1. Under a fixed northern angle of $30^{\circ}$, we move the plane PQ by letting $x_{Q}$ vary between 0.45 and 0.85 . The polyhedra $\Upsilon_{3}, \Upsilon_{4}$, and $\Upsilon_{5}$ are obtained as particular cases.

2. With fixed $x_{Q}=0.50$, we vary the northern angle by letting $y_{Q}$ range between 0.25 and 0.70 . We recover the cases of $\Upsilon_{1}$ and $\Upsilon_{3}$.

3. Under a fixed northern angle of $45^{\circ}$, we move the plane PQ by varying $x_{Q}$ between 0.35 and 0.65 . We recover the case of $\Upsilon_{1}$.

Complementarily we move the edge $\mathrm{Q}$ in steps of 0.05 in the $x$ - and $y$-direction.

All these computations were done using relatively coarse meshes, to keep the size of the system of linear equations below 6000. In each case we determined fifty eigenvalues.

\section{TABULATION OF RESULTS}

\subsection{Eigenvalues for Fundamental Domains of Groups}

Tables 1-5 display the eigenvalues we have found for the fundamental domains $\Upsilon_{1}$ to $\Upsilon_{5}$ of Section 3. The last digit given is always somewhat uncertain.

Having divided the set of eigenfunctions according to the symmetry types of the functions, we find that within each symmetry type the eigenfunctions are all simple. But it seems that the eigenfunctions belonging to specific symmetry types are included amongst the eigenvalues of another type. For example, in Table 1, type $G$ eigenvalues occur also under type D. This has to do with the existence of certain overgroups of the groups considered here.

\subsection{Eigenvalues for the Family of Tetrahedra}

Figures 5-7 display our results for the series of computations described in Section 7.2. In each case, we join the points corresponding to the $i$-th smallest eigenvalue as the parameter varies. 


\begin{tabular}{|ll|ll|ll|ll|ll|ll|ll|ll|}
\hline 44.85247 & $\mathrm{C}$ & 224.577 & $\mathrm{C}$ & 317.1 & $\mathrm{D}$ & 401.5 & $\mathrm{D}$ & 460.9 & $\mathrm{C}$ & 553.2 & $\mathrm{C}$ & 598 & $\mathrm{H}$ & 665.9 & $\mathrm{C}$ \\
74.1927 & $\mathrm{D}$ & 224.58 & $\mathrm{H}$ & 320.12 & $\mathrm{C}$ & 413.14 & $\mathrm{C}$ & 477.8 & $\mathrm{C}$ & 553.5 & $\mathrm{H}$ & 599 & $\mathrm{D}$ & 666 & $\mathrm{D}$ \\
104.649 & $\mathrm{C}$ & 236.60 & $\mathrm{D}$ & 320.12 & $\mathrm{H}$ & 413.3 & $\mathrm{H}$ & 477.8 & $\mathrm{H}$ & 556 & $\mathrm{D}$ & 609 & $\mathrm{D}$ & 667 & $\mathrm{H}$ \\
124.403 & $\mathrm{D}$ & 253.59 & $\mathrm{D}$ & 333.85 & $\mathrm{C}$ & 425.67 & $\mathrm{C}$ & 491.7 & $\mathrm{D}$ & 556 & $\mathrm{D}$ & 613 & $\mathrm{D}$ & 673.2 & $\mathrm{G}$ \\
147.781 & $\mathrm{C}$ & 263.69 & $\mathrm{C}$ & 355.70 & $\mathrm{C}$ & 429.9 & $\mathrm{G}$ & 511.2 & $\mathrm{C}$ & 569.7 & $\mathrm{C}$ & 627.8 & $\mathrm{C}$ & 679.4 & $\mathrm{C}$ \\
147.782 & $\mathrm{H}$ & 289.84 & $\mathrm{C}$ & 364.8 & $\mathrm{D}$ & 430.6 & $\mathrm{D}$ & 511.6 & $\mathrm{H}$ & 571.8 & $\mathrm{G}$ & 628 & $\mathrm{H}$ & 680 & $\mathrm{D}$ \\
166.640 & $\mathrm{D}$ & 289.87 & $\mathrm{H}$ & 370.9 & $\mathrm{D}$ & 434.6 & $\mathrm{D}$ & 515.2 & $\mathrm{D}$ & 572.3 & $\mathrm{C}$ & 648.0 & $\mathrm{C}$ & 683.8 & $\mathrm{C}$ \\
166.880 & $\mathrm{C}$ & 301.60 & $\mathrm{D}$ & 378.1 & $\mathrm{D}$ & 458.2 & $\mathrm{C}$ & 523.5 & $\mathrm{C}$ & 572.4 & $\mathrm{H}$ & 651 & $\mathrm{D}$ & 684 & $\mathrm{H}$ \\
199.25 & $\mathrm{D}$ & 305.57 & $\mathrm{G}$ & 378.58 & $\mathrm{C}$ & 458.3 & $\mathrm{H}$ & 528.5 & $\mathrm{G}$ & 576 & $\mathrm{D}$ & 655 & $\mathrm{D}$ & 697.7 & $\mathrm{C}$ \\
201.179 & $\mathrm{C}$ & 305.7 & $\mathrm{D}$ & 378.6 & $\mathrm{H}$ & 460.5 & $\mathrm{D}$ & 530 & $\mathrm{D}$ & 597.5 & $\mathrm{C}$ & 663.2 & $\mathrm{G}$ & 703 & $\mathrm{D}$ \\
\hline
\end{tabular}

TABLE 1. Eigenvalues up to 703 for $\Upsilon_{1}$, the fundamental domain of $\mathrm{PSL}_{2}(\mathbb{Z}[i])$, and symmetry type of their eigenfunctions. The entries of type $\mathrm{D}$ are also the eigenvalues for $\Theta_{1}$, corresponding to the extended Bianchi group $\mathrm{EB}(\mathbb{Z}[i])$. Types $\mathrm{C}$ and $\mathrm{D}$ together constitute the eigenvalues for $\mathrm{PGL}_{2}(\mathbb{Z}[i])$. In this and subsequent tables, the last digit of each entry may be untrustworthy.

\begin{tabular}{|cl|ll|ll|ll|ll|ll|ll|ll|cc|}
\hline 25.4420 & $\mathrm{P}$ & 107.71 & $\mathrm{D}$ & 165.9 & $\mathrm{H}$ & 201.5 & $\mathrm{P}$ & 250.2 & $\mathrm{P}$ & 282.6 & $\mathrm{P}$ & 314 & $\mathrm{H}$ & 341 & $\mathrm{P}$ & 374 & $\mathrm{H}$ \\
28.471 & $\mathrm{D}$ & 111.23 & $\mathrm{H}$ & 166.5 & $\mathrm{D}$ & 211.5 & $\mathrm{P}$ & 255.4 & $\mathrm{~T}$ & 283.8 & $\mathrm{D}$ & 316 & $\mathrm{D}$ & 347 & $\mathrm{D}$ & 376 & $\mathrm{~T}$ \\
45.097 & $\mathrm{D}$ & 111.36 & $\mathrm{D}$ & 168.5 & $\mathrm{P}$ & 212.5 & $\mathrm{D}$ & 257.3 & $\mathrm{P}$ & 285.2 & $\mathrm{~T}$ & 317 & $\mathrm{D}$ & 350 & $\mathrm{D}$ & 377 & $\mathrm{D}$ \\
55.056 & $\mathrm{P}$ & 120.01 & $\mathrm{D}$ & 174.2 & $\mathrm{D}$ & 213.5 & $\mathrm{~T}$ & 257.8 & $\mathrm{D}$ & 286.0 & $\mathrm{P}$ & 317 & $\mathrm{D}$ & 350 & $\mathrm{H}$ & 377 & $\mathrm{~T}$ \\
63.150 & $\mathrm{D}$ & 132.66 & $\mathrm{~T}$ & 182.9 & $\mathrm{D}$ & 213.6 & $\mathrm{P}$ & 258.2 & $\mathrm{H}$ & 292.7 & $\mathrm{P}$ & 320 & $\mathrm{~T}$ & 352 & $\mathrm{D}$ & 378 & $\mathrm{P}$ \\
70.205 & $\mathrm{P}$ & 132.75 & $\mathrm{P}$ & 189.2 & $\mathrm{~T}$ & 214.9 & $\mathrm{D}$ & 259.5 & $\mathrm{D}$ & 295 & $\mathrm{P}$ & 322 & $\mathrm{P}$ & 362 & $\mathrm{D}$ & 380 & $\mathrm{P}$ \\
77.40 & $\mathrm{~T}$ & 134.13 & $\mathrm{P}$ & 189.4 & $\mathrm{P}$ & 226.7 & $\mathrm{P}$ & 260.2 & $\mathrm{H}$ & 301 & $\mathrm{~T}$ & 327 & $\mathrm{D}$ & 362 & $\mathrm{~T}$ & 383 & $\mathrm{D}$ \\
77.45 & $\mathrm{P}$ & 136.18 & $\mathrm{D}$ & 189.9 & $\mathrm{~T}$ & 228.6 & $\mathrm{D}$ & 261.1 & $\mathrm{D}$ & 302 & $\mathrm{P}$ & 329 & $\mathrm{~T}$ & 363 & $\mathrm{P}$ & 384 & $\mathrm{~T}$ \\
79.25 & $\mathrm{D}$ & 143.36 & $\mathrm{D}$ & 190.3 & $\mathrm{P}$ & 229.7 & $\mathrm{H}$ & 264.8 & $\mathrm{~T}$ & 303 & $\mathrm{D}$ & 332 & $\mathrm{~T}$ & 364 & $\mathrm{P}$ & 386 & $\mathrm{D}$ \\
95.78 & $\mathrm{D}$ & 150.26 & $\mathrm{~T}$ & 192.3 & $\mathrm{D}$ & 231.0 & $\mathrm{D}$ & 265.0 & $\mathrm{P}$ & 309 & $\mathrm{H}$ & 333 & $\mathrm{P}$ & 367 & $\mathrm{D}$ & 388 & $\mathrm{P}$ \\
104.25 & $\mathrm{~T}$ & 150.33 & $\mathrm{P}$ & 195.2 & $\mathrm{H}$ & 239.9 & $\mathrm{~T}$ & 266.1 & $\mathrm{D}$ & 310 & $\mathrm{D}$ & 334 & $\mathrm{P}$ & 367 & $\mathrm{H}$ & 392 & $\mathrm{D}$ \\
104.31 & $\mathrm{P}$ & 156.0 & $\mathrm{D}$ & 195.3 & $\mathrm{D}$ & 240.1 & $\mathrm{P}$ & 279.4 & $\mathrm{D}$ & 312 & $\mathrm{H}$ & 334 & $\mathrm{~T}$ & 371 & $\mathrm{P}$ & & \\
107.45 & $\mathrm{P}$ & 160.9 & $\mathrm{P}$ & 201.0 & $\mathrm{~T}$ & 245.3 & $\mathrm{D}$ & 281.5 & $\mathrm{~T}$ & 314 & $\mathrm{D}$ & 335 & $\mathrm{P}$ & 373 & $\mathrm{D}$ & \\
\hline
\end{tabular}

TABLE 2. Eigenvalues up to 392 for $\Upsilon_{2}$, the fundamental domain of $\mathrm{PSL}_{2}(\mathbb{Z}[\sqrt{-2}])$. The entries of type $\mathrm{D}$ are also the eigenvalues for $\Theta_{2}$, corresponding to the group $\operatorname{EB}(\mathbb{Z}[\sqrt{-2}])$.

\begin{tabular}{|cl|cc|cc|cc|cc|cc|cc|cc|cc|}
\hline 51.014 & $\mathrm{~B}$ & 222.0 & $\mathrm{~B}$ & 293.5 & $\mathrm{D}$ & 365.1 & $\mathrm{D}$ & 441 & $\mathrm{~B}$ & 484 & $\mathrm{~B}$ & 515 & $\mathrm{G}$ & 568 & $\mathrm{D}$ & 605 & $\mathrm{~B}$ \\
122.19 & $\mathrm{~B}$ & 226.4 & $\mathrm{D}$ & 304.1 & $\mathrm{~B}$ & 375.7 & $\mathrm{~J}$ & 446 & $\mathrm{D}$ & 498 & $\mathrm{~B}$ & 544 & $\mathrm{~B}$ & 596 & $\mathrm{D}$ & 642 & $\mathrm{~B}$ \\
157.29 & $\mathrm{D}$ & 261.5 & $\mathrm{~J}$ & 331.2 & $\mathrm{~B}$ & 376.0 & $\mathrm{~B}$ & 450 & $\mathrm{D}$ & 514 & $\mathrm{D}$ & 544 & $\mathrm{~J}$ & 597 & $\mathrm{D}$ & 665 & $\mathrm{~B}$ \\
177.78 & $\mathrm{~B}$ & 261.6 & $\mathrm{~B}$ & 355.9 & $\mathrm{D}$ & 408.7 & $\mathrm{~B}$ & 483 & $\mathrm{~J}$ & 515 & $\mathrm{D}$ & 553 & $\mathrm{~B}$ & 602 & $\mathrm{~J}$ & 669 & $\mathrm{D}$ \\
\hline
\end{tabular}

TABLE 3. Eigenvalues up to 675 for $\Upsilon_{3}$, the fundamental domain of $\mathrm{PSL}_{2}(\mathbb{Z}[\omega])$. The entries of type $\mathrm{D}$ are also the eigenvalues for $\Theta_{3}$, corresponding to the group $\operatorname{EB}(\mathbb{Z}[\omega])$. 


\begin{tabular}{|c|c|c|c|c|c|c|c|c|}
\hline $16.490 \mathrm{~B}$ & $91.11 \mathrm{D}$ & $135.88 \mathrm{~J}$ & 160.0 & 205.15 & $232.7 \mathrm{~B}$ & 256.8 B & $293.5 \mathrm{D}$ & $301.9 \mathrm{D}$ \\
\hline $45.856 \mathrm{D}$ & $100.77 \mathrm{D}$ & $135.91 \mathrm{~B}$ & $177.78 \quad \mathrm{~B}$ & 205.2 & $238.1 \mathrm{D}$ & $261.5 \mathrm{~J}$ & 294.9 B & 304.1 B \\
\hline $51.014 \mathrm{~B}$ & $107.14 \mathrm{~B}$ & $139.77 \mathrm{D}$ & $185.2 \quad \mathrm{D}$ & 222.0 & $248.3 \mathrm{G}$ & $261.6 \mathrm{~B}$ & $299.2 \mathrm{~J}$ & \\
\hline $78.41 \quad \mathrm{~B}$ & $122.19 \mathrm{~B}$ & $157.29 \mathrm{D}$ & 198.7 & 226.4 & $248.4 \mathrm{D}$ & $270.2 \mathrm{D}$ & $299.8 \mathrm{~B}$ & \\
\hline
\end{tabular}

TABLE 4. Eigenvalues up to 305 for $\Upsilon_{4}$, the fundamental domain of the group $\Gamma_{4}$ of Section 3.5. The entries of type $\mathrm{D}$ are also the eigenvalues for $\Theta_{4}$, corresponding to the group $E_{4}$.

\begin{tabular}{|ll|ll|cl|ll|lllll|ll|ll|}
\hline 7.322 & $\mathrm{~B}$ & 63.7 & $\mathrm{~B}$ & 95 & $\mathrm{D}$ & 118 & $\mathrm{D}$ & 151.4 & $\mathrm{~B}$ & 170 & $\mathrm{~B}$ & 189 & $\mathrm{D}$ & 199.7 & $\mathrm{~J}$ \\
24.43 & $\mathrm{~B}$ & 74.6 & $\mathrm{~B}$ & 103.3 & $\mathrm{~J}$ & 128 & $\mathrm{~B}$ & 156.7 & $\mathrm{~J}$ & 173 & $\mathrm{D}$ & 192 & $\mathrm{~B}$ & 212 & $\mathrm{~B}$ \\
43.5 & $\mathrm{~B}$ & 87.0 & $\mathrm{~B}$ & 112 & $\mathrm{D}$ & 145 & $\mathrm{D}$ & 163 & $\mathrm{D}$ & 179.6 & $\mathrm{G}$ & 196 & $\mathrm{D}$ & $216 ?$ & $\mathrm{D}$ \\
45.1 & $\mathrm{D}$ & 93.6 & $\mathrm{~B}$ & 116 & $\mathrm{~B}$ & 145.5 & $\mathrm{~B}$ & 166.3 & $\mathrm{~B}$ & 185.9 & $\mathrm{~B}$ & 199 & $\mathrm{D}$ & 220 & $\mathrm{~B}$ \\
\hline
\end{tabular}

TABLE 5. Eigenvalues up to 220 for $\Upsilon_{5}$, the fundamental domain of the group $\Gamma_{5}$ of Section 3.6. The entries of type $\mathrm{D}$ are also the eigenvalues for $\Theta_{5}$, corresponding to the group $E_{5}$. The question mark indicates we were not certain from the numerical results that the eigenfunction we obtained for this eigenvalue satisfied the $L^{2}$-condition.
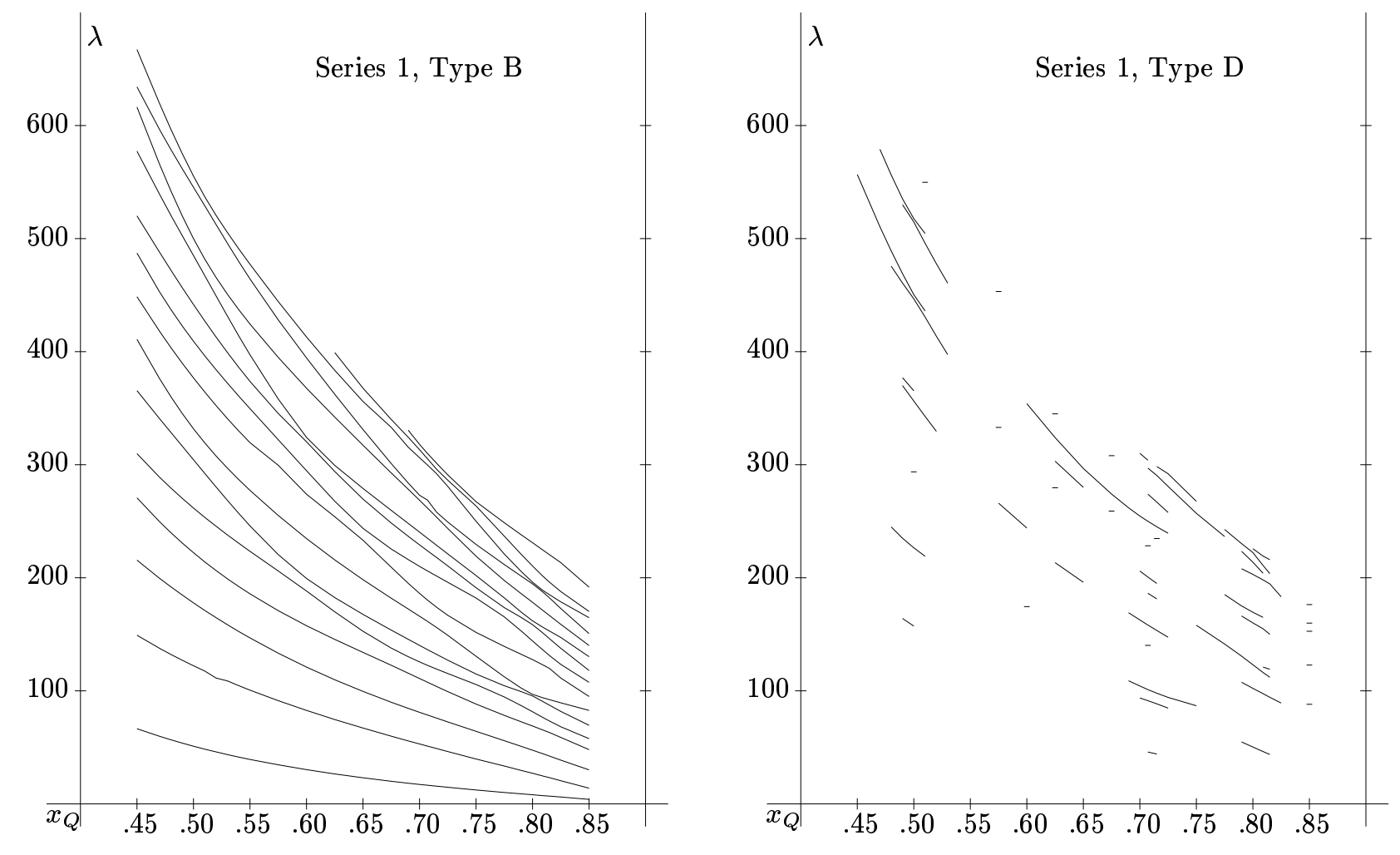

FIGURE 5. Eigenvalues from series 1, types B and D. 

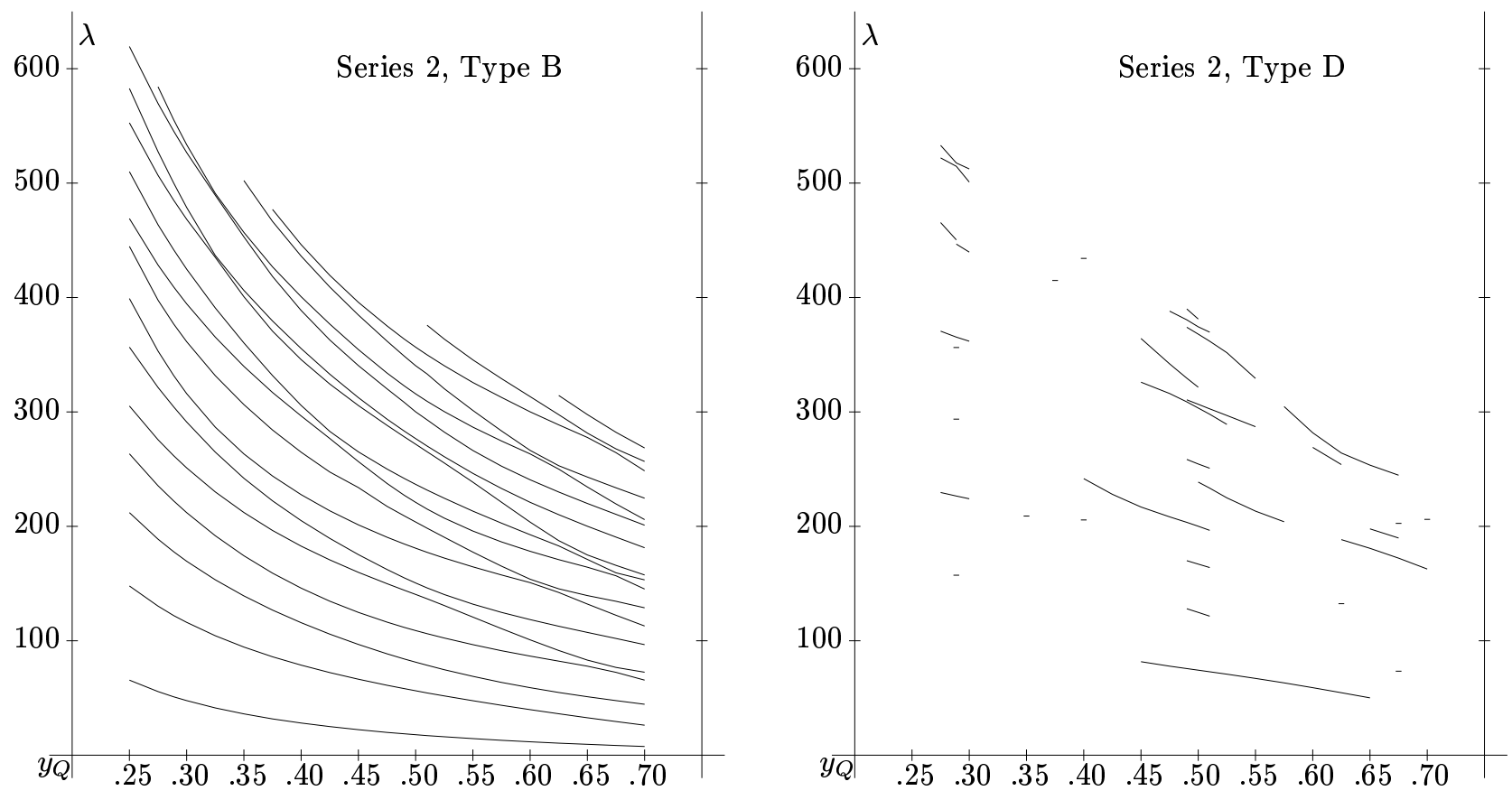

FIGURE 6. Eigenvalues from series 2, types B and D.
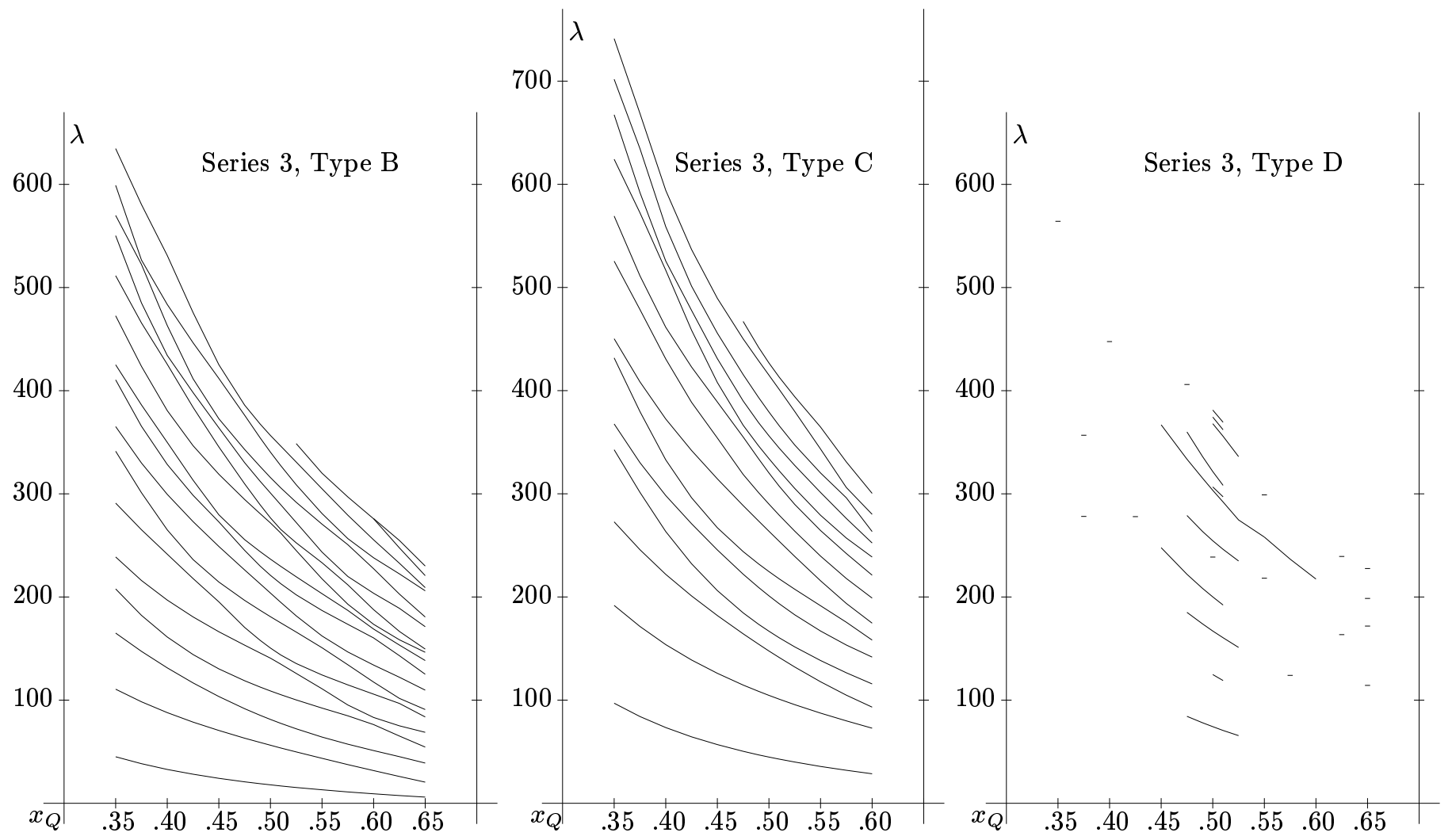

FIGURE 7. Eigenvalues from series 3, types B, C, and D. 
Eigenvalue count (Type D, good)

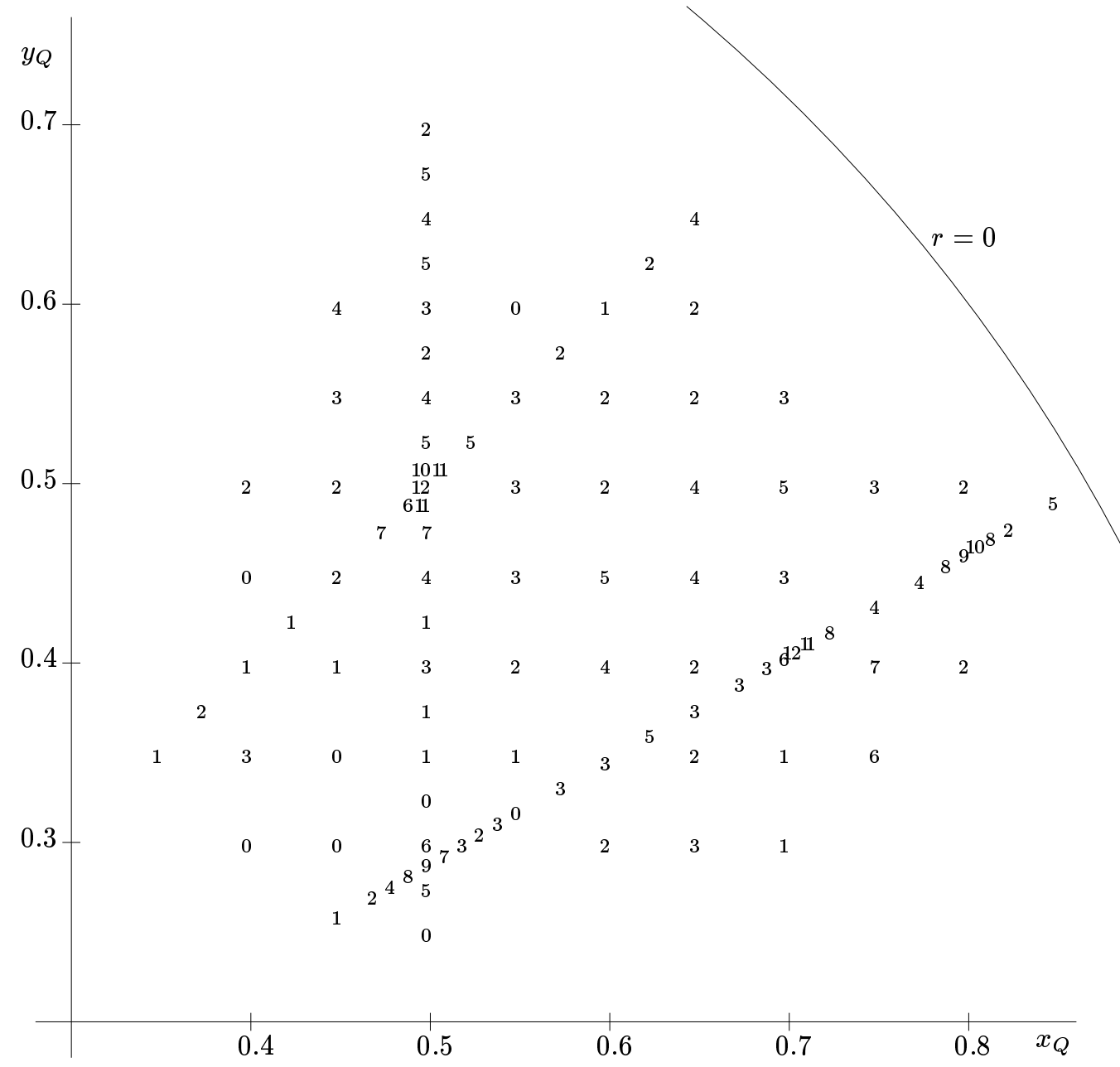

FIGURE 8. Number of eigenvalues of type $D$ found for different tetrahedra $\Theta\left(x_{Q}, y_{Q}\right)$. In this figure we only count the eigenvalues for which the quality of the approximation for the iterated eigenfunction was very good. Compare Figure 9.

Eigenvalues of types B and C vary continuously with the parameters. This is expected from the minimax principle, since the spectrum is purely discrete because of the Dirichlet boundary conditions. The curves descend as the parameter grows, since the volume of the tetrahedron grows.

For eigenvalues of Type D-those subject to full Neumann boundary conditions - the case is altered. As the figures show, the behavior here is erratic: eigenvalues seem to come and go. Of course an eigenvalue existing on one tetrahedron may affect heavily the computation on neighboring tetrahedra, so the little pieces of curves may only be points.
For certain values of the parameter, namely, for $x_{Q}=0.5$ and $x_{Q} \approx 0.707$ in series 1 , for $y_{Q} \approx 0.289$ and $y_{Q}=0.5$ in series 2 , and for $x_{Q}=0.5$ in series 3 , we pass over the fundamental domain of an arithmetic congruence group, and we expectedly find many pieces of curves near these parameter values. We also find many pieces of curves for $x_{Q} \approx 0.809$ in series 1 ; this corresponds to the fundamental domain of the nonarithmetic group $E_{5}$.

Another way to look at the data for eigenvalues of type $\mathrm{D}$ is shown in Figures 8 and 9. Recall that the tetrahedron $\Theta\left(x_{Q}, y_{Q}\right)$ has its lower vertices above the points $(0,0),\left(x_{Q}, 0\right)$, and $\left(x_{Q}, y_{Q}\right)$. For 
Eigenvalue count (Type D, good and dubious)

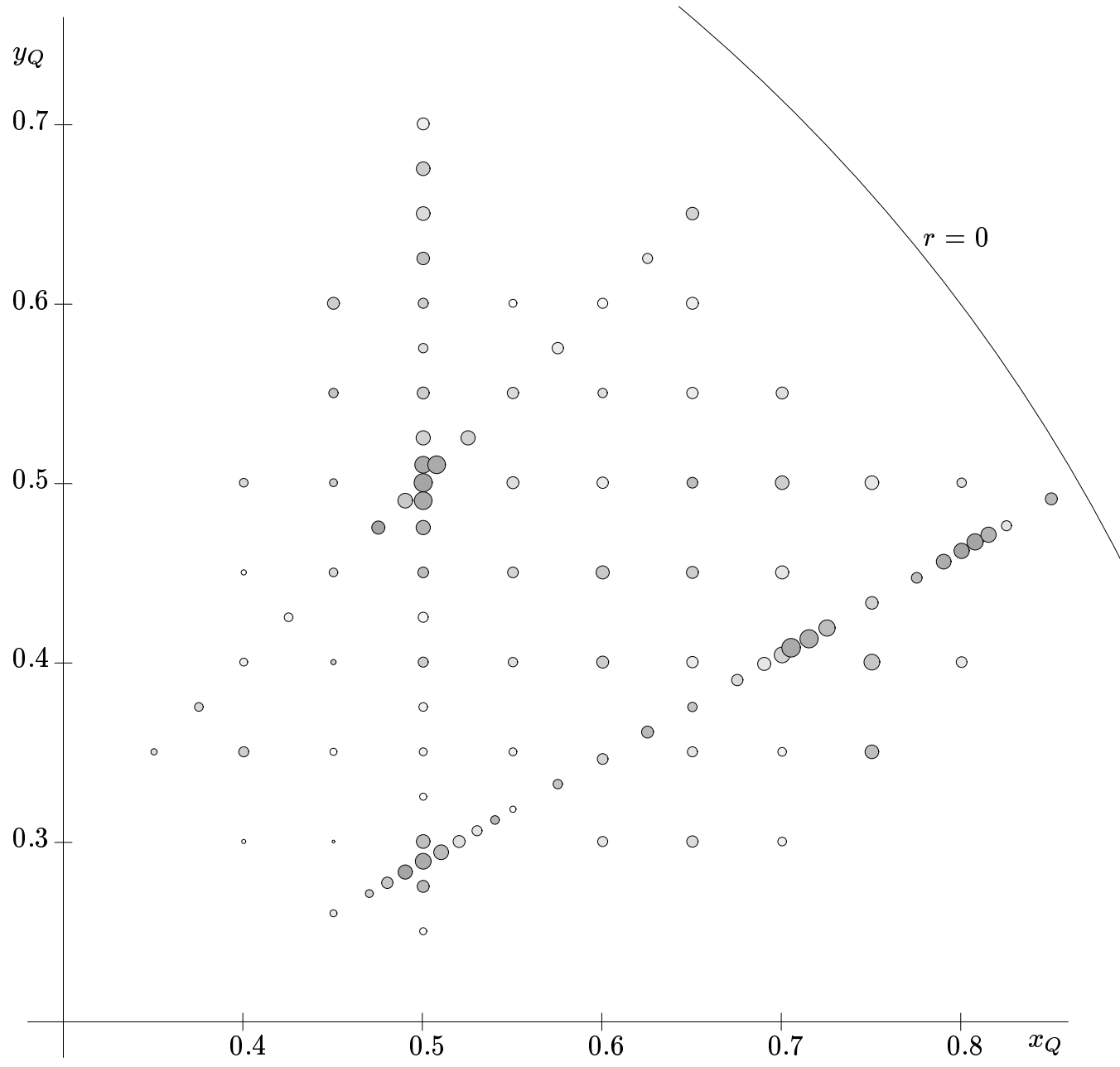

FIGURE 9. Number of eigenvalues of type $\mathrm{D}$ found for different tetrahedra $\Theta\left(x_{Q}, y_{Q}\right)$, as in Figure 8 , but including dubious eigenvalues (those for which the eigenfunction approximation was not very good). The area of each disk is proportional to the total number of eigenvalues, and the darkness increases with the proportion of good eigenvalues. For calibration, the disk $\bigcirc$ at $\left(\frac{1}{2}, \frac{1}{2}\right)$ represents 12 good and 12 dubious eigenvalues.

different values of $\left(x_{Q}, y_{Q}\right)$, we have placed at the point $\left(x_{Q}, y_{Q}\right)$ the number of eigenvalues of type $\mathrm{D}$ for $\Theta\left(x_{Q}, y_{Q}\right)$. In both figures the local maxima are found where a fundamental domain is passed. This may indicate that only tesselating tetrahedra can carry eigenfunctions of type D for the Laplace operator.

\section{REFERENCES}

[Abramowitz and Stegun 1965] M. Abramowitz, I. A. Stegun (editors), Handbook of Mathematical Functions, Dover, New York 1965.
[Axelsson and Barker 1984] O. Axelsson, V. A. Barker, Finite Element Solution of Boundary Value Problems: Theory and Computation, Academic Press, Orlando, Florida, 1984.

[Babuška 1986] I. Babuška, "Feedback, adaptivity, and a-posteriori estimates in finite elements: aims, theory, and experience", pp. 3-23 in Accuracy Estimates and Adaptive Refinements in Finite Element Computations, Lisbon, 1984 (edited by I. Babuška et al.), Wiley, Chicester, 1986.

[Babuška 1988] I. Babuška, "Advances in the $p$ and $h$ $p$ versions of the finite element method. A survey", 
pp. 31-46 in Numerical Mathematics, Singapore, 1988 (edited by R. P. Agarval et al.), Internat. Schriftenreihe Numer. Math. 86, Birkhäuser, Basel, 1988.

[Babuška and Aziz 1976] I. Babuška and A. K. Aziz, "On the angle condition in the finite element method", SIAM J. Numer. Anal. 13 (1976), 214-226.

[Babuška and Guo 1988] I. Babuška and B. Q. Guo, "Regularity of the solution of elliptic problems with piecewise analytic data. I. Boundary value problems for linear elliptic equations of second order", SIAM J. Math. Anal. 19 (1988), 172-203.

[Babuška and Guo 1992] I. Babuška and B. Q. Guo, "The $h, p$ and $h-p$ version of the finite element method; basic theory and applications", Advances in Engineering Software 15 (1992), 159-172.

[Babuška et al. 1989] I. Babuška, B. Q. Guo, and J. E. Osborn, "Regularity and numerical solution of eigenvalue problems with piecewise analytic data", SIAM J. Numer. Anal. 26 (1989), 1534-1560.

[Babuška and Osborn 1987] I. Babuška and J. E. Osborn, "Estimates for the errors in eigenvalue and eigenvector approximation by Galerkin methods, with particular attention to the case of multiple eigenvalues", SIAM J. Numer. Anal. 24 (1987), 1249-1276.

[Babuška and Osborn 1989] I. Babuška and J. E. Osborn, "Finite element-Galerkin approximation of the eigenvalues and eigenvectors of selfadjoint problems", Math. Comp. 52 (1989), 275-297.

[Babuška and Osborn 1991] I. Babuška and J. E. Osborn, "Eigenvalue problems", pp. 641-787 in Handbook of Numerical Analysis, II: Finite Element Methods, Part 1 (edited by P. G. Ciarlet and J. L. Lions), North-Holland, Amsterdam, 1991.

[Banerjee 1992] U. Banerjee, "A note on the effect of numerical quadrature in finite element eigenvalue approximation", Numer. Math. 61 (1992), 145-152.

[Banerjee and Osborn 1990] U. Banerjee and J. E. Osborn, "Estimation of the effect of numerical integration in finite element eigenvalue approximation", $\mathrm{Nu}$ mer. Math. 56 (1990), 735-762.

[Bank 1983] R. E. Bank, "The efficient implementation of local mesh refinement algorithms", pp. 74-81 in
Adaptive Computational Methods for Partial Differential Equations (edited by I. Babuška et al.), SIAM, Philadelphia 1983.

[Bank and Sherman 1979] R. E. Bank and A. H. Sherman, "The use of adaptive grid refinement for badly behaved elliptic partial differential equations", pp. 33-39 in Advances in Computer Methods for Partial Differential Equations III, Bethlehem, PA, 1979 (edited by R. Vichnevetsky and R. S. Stepleman), IMACS, New Brunswick, NJ, 1979. Reprinted as pp. 18-24 in Math. Comput. Simulation 22.

[Bank and Sherman 1981] R. E. Bank and A. H. Sherman, "An adaptive multilevel method for elliptic boundary problems", Computing 26 (1981), 91-105.

[Bank et al. 1983] R. E. Bank, A. H. Sherman, and A. Weiser, "Refinement algorithms and data structures for regular local mesh refinement", pp. 317 in Scientific computing, Montreal, 1982 (edited by R. S. Stepleman), IMACS, New Brunswick, NJ, and North-Holland, Amsterdam, 1983.

[Bianchi 1892] L. Bianchi, "Sui gruppi de sostituzioni lineari con coefficienti appartenenti a corpi quadratici immaginari", Math. Ann. 40 (1892), 332-412.

[Bogomolny et al. 1992] E. Bogomolny, B. Georgot, M.-J. Giannoni, and C. Schmit, "Chaotic billiards generated by arithmetic groups", Phys. Rev. Lett. 69 (1992), 1477-1480.

[Bolte et al. 1992] J. Bolte, G. Steil, and F. Steiner, "Arithmetical chaos and violation of universality in energy level statistics", Phys. Rev. Lett. 69 (1992), 2188-2191.

[Chatellin 1983] F. Chatellin, Spectral Approximation of Linear Operators, Academic Press, New York, 1983.

[Ciarlet 1978] P. G. Ciarlet, The Finite Element Method for Elliptic Problems, North-Holland, Amsterdam, 1978.

[Ciarlet and Raviart 1972] P. G. Ciarlet and P.-A. Raviart, "The combined effect of curved boundaries and numerical integration in isoparametric finite element methods", pp. 409-474 in The Mathematical Foundations of the Finite Element Method with Applications to Partial Differential Equations (edited by A. K. Aziz), Academic Press, New York, 1972. 
[Concus et al. 1976] P. Concus, G. H. Golub, and D. P. O'Leary, “A generalized conjugate gradient method for the numerical solution of elliptic partial differential equations", pp. 309-332 in Sparse Matrix Computations (edited by J. R. Bunch and D. J. Rose), Academic Press, New York, 1976.

[Elstrodt et al. 1985] J. Elstrodt, F. Grunewald, and J. Mennicke, "Eisenstein series on three-dimensional hyperbolic space and imaginary quadratic number fields", J. reine angew. Math. 360 (1985), 160-213.

[Elstrodt et al. 1987] J. Elstrodt, F. Grunewald and J. Mennicke, "Some remarks on discrete subgroups of $\mathrm{SL}_{2}(\mathbb{C})$ (Russian), Zapiski Nauchnykh Seminarov Mat. Inst. Steklov. 162 (1987), 77-106. English translation in J. Sov. Math. 46 (1989), 1760-1788.

[Elstrodt et al. $\geq 1996]$ J. Elstrodt, F. Grunewald, and J. Mennicke, "Groups acting on 3-dimensional hyperbolic space", in preparation.

[Ergatoudis et al. 1968] J. G. Ergatoudis, B. M. Irons, and O. C. Zienkiewicz, "Curved, isoparametric, quadrilateral elements for finite element analysis", Internat. J. Solids Structures 4 (1968), 31-42.

[Ewing 1990] R. E. Ewing, "A posteriori error estimation", Computer Methods in Applied Mechanics and Engineering 82 (1990), 59-72.

[Gambolati and Putti 1994] G. Gambolati and M. Putti, "A comparison of Lanczos and optimization methods in the partial solution of sparse symmetric eigenproblems", Internat. J. Numer. Methods Engrg. 37 (1994), 605-621.

[Grunewald and Huntebrinker] F. Grunewald and W. Huntebrinker, "A numerical study of eigenvalues of the hyperbolic Laplacian for polyhedra with two cusps", in preparation.

[Hackbusch 1986] W. Hackbusch, Theorie und $\mathrm{Nu}$ merik elliptischer Differentialgleichungen, Teubner, Stuttgart, 1986.

[Hackbusch 1993] W. Hackbusch, Iterative Lösung großer schwachbesetzter Gleichungssysteme, 2. Auflage, Teubner, Stuttgart, 1993. Translated as Iterative Solution of Large Sparse Systems of Equations, Springer, New York, 1994.

[Hejhal 1983] D. A. Hejhal, The Selberg Trace Formula for $\operatorname{PSL}(2, \mathbb{R})$, Vol. 2, Lecture Notes in Mathematics 1001, Springer, Berlin 1983.
[Hejhal 1992] D. A. Hejhal, "Eigenvalues of the Laplacian for Hecke Triangle Groups", Mem. Amer. Math. Soc. 97 (1992), no. 469, 165 pp.

[Hejhal and Rackner 1992] D. A. Hejhal and B. Rackner, "On the topography of Maass Waveforms for $\operatorname{PSL}(2, \mathbb{Z})$ : experiments and heuristics", Experimental Math. 1 (1992), 275-305.

[Hestenes and Stiefel 1952] M. R. Hestenes, E. Stiefel, "Methods of conjugate gradients for solving linear systems", J. Res. Nat. Bur. Standards 49 (1952), $409-436$.

[Hughes 1987] T. J. R. Hughes, The Finite Element Method: Linear Static and Dynamic Finite Element Analysis, Prentice-Hall, Englewood Cliffs, N. J., 1987.

[Huntebrinker 1991] W. Huntebrinker, "Numerische Bestimmung von Eigenwerten des Laplace-Operators auf hyperbolischen Räumen mit adaptiven FiniteElement-Methoden", Bonner Math. Schriften 225 (1991).

[Huntebrinker 1995] W. Huntebrinker, "Numerische Bestimmung von Eigenwerten des Laplace-BeltramiOperators auf dreidimensionalen hyperbolischen Räumen mit Finite-Element-Methoden", Dissertation, Heinrich-Heine-Universität Düsseldorf, 1995.

[Jamet 1976] P. Jamet, "Estimations de l'erreur pour les éléments finis droits presque dégénérés", RAIRO Anal. Numér. 10 (1976), 43-60.

[Krizek 1992] M. Krizek, "On the maximum angle condition for linear tetrahedral elements", SIAM Journal on Numerical Analysis 29 (1992), 513-520.

[Maaß 1949a] H. Maaß, "Über eine neue Art von nichtanalytischen automorphen Funktionen und die Bestimmung Dirichletscher Reihen durch Funktionalgleichungen", Math. Ann. 121 (1949), 141-183.

[Maaß 1949b] H. Maaß, "Automorphe Funktionen in mehreren Veränderlichen und Dirichletsche Reihen", Abh. Math. Sem. Univ. Hamburg 16 (1949), 72-100.

[Phillips and Sarnak 1985a] R. S. Phillips and P. Sarnak, "On cusp forms for co-finite subgroups of $\operatorname{PSL}(2, \mathbb{R}) "$, Invent. Math. 80 (1985), 339-364.

[Phillips and Sarnak 1985b] R. S. Phillips and P. Sarnak, "The Weyl theorem and the deformation 
of discrete groups", Comm. Pure Appl. Math. 38 (1985), 853-866.

[Picard 1884] E. Picard, "Sur un groupe de transformations des points de l'espace situés du même côté d'un plan", Bull. Soc. Math. France 12 (1884), 43-47.

[Sarnak 1986] P. Sarnak, "On cusp forms", pp. 393407 in The Selberg Trace Formula and Related Topics (edited by D. A. Hejhal et al.), Contemporary Mathematics 53, Amer. Math. Soc., Providence, RI, 1986.

[Sarnak 1990] P. Sarnak, "On cusp forms II", pp. 237250 in Festschrift in honour of I. I. PiatetskiShapiro, Part II (edited by S. Gelbart et al.), Israel Mathematical Conference Proceedings 3, Weizmann Institute, Jerusalem, 1990.

[Sartoletto et al. 1989] F. Sartoletto, G. Pini, and G. Gambolati, "Accelerated simultaneous iterations for large finite element eigenproblems", J. Comput. Phys. 81 (1989), 53-69.

[Schwarz 1991] H. R. Schwarz, Methode der finiten Elemente, 3. Auflage, Teubner, Stuttgart, 1991. Translated as Finite Element Methods, Academic Press, London 1988.

[Selberg 1956] A. Selberg, "Harmonic analysis and discontinuous groups in weakly symmetric Riemann spaces with applications to Dirichlet series", J. Indian Math. Soc. (New Series) 20 (1956), 47-87.

[Smotrov and Golovchansky 1991] M. N. Smotrov and V. V. Golovchansky, "Small eigenvalues of the Laplacian on $\Gamma \backslash H_{3}$ for $\Gamma=\mathrm{PSL}_{2}(\mathbb{Z}[i])$ ", Preprint 91-040, Universität Bielefeld, SBF 343, 1991.

[Steil 1994] G. Steil: "Eigenvalues of the Laplacian and of the Hecke operators for $\operatorname{PSL}(2, \mathbb{Z})$ ", Preprint 94028, Institut für Theoretische Physik der Universität Hamburg. Submitted to Math. Comp.

[Stramm 1994] K. Stramm, "Kleine Eigenwerte des Laplace-Operators zu Kongruenzgruppen", Schriftenreihe des Mathematischen Instituts und des Graduiertenkollegs der Universität Münster, 3. Serie 11 (1994).

[Strouboulis and Haque 1992] T. Strouboulis and K. A. Haque, "Recent experiences with error estimation and adaptivity", Part I: "Review of error estimators for scalar elliptic problems" and Part II: "Error estimation for $h$-adaptive approximations on grids of triangles und quadrilaterals", Comput. Methods Appl. Mech. Engrg. 97 (1992), 399-436 and 100 (1992), 359-430.

[Swan 1971] R. G. Swan, "Generators and relations for certain special linear groups", Adv. Math. 6 (1971), $1-77$.

[Venkov 1977a] A. B. Venkov, "On an asymptotic formula connected with the number of eigenvalues corresponding to odd eigenfunctions of the LaplaceBeltrami operator on a fundamental region of the modular group PSL(2, Z)" (Russian), Dokl. Akad. Nauk SSSR 233 (1977), 1021-1023. Translated in Soviet Math. Doklady 18 (1977), 524-526).

[Venkov 1977b] A. B. Venkov, "On the space of cusp forms for certain Fuchsian groups generated by reflections" (Russian), Dokl. Akad. Nauk SSSR 236 (1977), 525-527. Translated in Soviet Math. Doklady 18 (1977), 1214-1217).

[Venkov 1978] A. B. Venkov, "On the space of cusp functions for a Fuchsian group of the first kind with nontrivial commensurator" (Russian), Dokl. Akad. Nauk SSSR 239 (1978), 511-514. Translated in Soviet Math. Doklady 19 (1978), 343-347).

[Venkov 1979] A. B. Venkov, "The Artin-Takagi formula for Selberg's Zeta-function and the Roelcke conjecture" (Russian), Dokl. Akad. Nauk SSSR 247 (1979), 540-542. Translated in Soviet Math. Doklady 20 (1979), 745-748.

[Venkov 1981] A. B. Venkov, "Spectral Theory of Automorphic Functions" (Russian), Trudy Mat. Inst. Steklov. 153 (1981), 172 pp. Translated in Proc. Steklov Inst. Math. 1982, no. 4, 163 pp. (1983).

[Watkins 1993] D. S. Watkins, "Some perspectives on the eigenvalue problem", SIAM Review 35 (1993), $430-471$.

[Wolpert 1994] S. A. Wolpert: "Disappearance of cusp forms in special families", Ann. Math. (2nd Series) 139 (1994), 239-291.

[Zienkiewicz and Morgan 1983] O. C. Zienkiewicz and K. Morgan, Finite Elements and Approximation, Wiley, Chicester, 1983.

[Zienkiewicz and Zhu 1991] O. C. Zienkiewicz and J. Z. Zhu, "Adaptivity and mesh generation", Internat. J. Numer. Methods Engrg. 32 (1991), 783-810. 
Fritz Grunewald, Mathematisches Institut, Heinrich-Heine-Universität Düsseldorf, Universitätsstraße 1, D-40225 Düsseldorf, Germany (fritz@math.uni-duesseldorf.de)

Wolfgang Huntebrinker, Mathematisches Institut, Heinrich-Heine-Universität Düsseldorf, Universitätsstraße 1, D-40225 Düsseldorf, Germany (whuntebr@math.uni-duesseldorf.de)

Received August 30, 1994; accepted in revised form October 10, 1995 